Article

\title{
The Rare IL22RA2 Signal Peptide Coding Variant rs28385692 Decreases Secretion of IL-22BP Isoform-1, -2 and -3 and Is Associated with Risk for Multiple Sclerosis
}

Paloma Gómez-Fernández ${ }^{1,+} \mathbb{D}$, Aitzkoa Lopez de Lapuente Portilla ${ }^{1,2,+}$, Ianire Astobiza ${ }^{1}$, Jorge Mena ${ }^{1,3}$, Andoni Urtasun ${ }^{1}$, Vivian Altmann ${ }^{4}(\mathbb{D})$, Fuencisla Matesanz ${ }^{5}$, David Otaegui ${ }^{6}(\mathbb{D}$, Elena Urcelay ${ }^{7}$, Alfredo Antigüedad ${ }^{8}$, Sunny Malhotra ${ }^{9}$, Xavier Montalban ${ }^{9}$, Tamara Castillo-Triviño ${ }^{6}$, Laura Espino-Paisán ${ }^{7}$, Orhan Aktas ${ }^{10} \mathbb{D}$, Mathias Buttmann ${ }^{11,12}(\mathbb{D}$, Andrew Chan ${ }^{13}$, Bertrand Fontaine ${ }^{14}$, Pierre-Antoine Gourraud ${ }^{15,16}$, Michael Hecker ${ }^{17}(\mathbb{D}$, Sabine Hoffjan ${ }^{18}$, Christian Kubisch ${ }^{19}$, Tania Kümpfel ${ }^{20}$, Felix Luessi ${ }^{21}$ (D), Uwe K. Zettl ${ }^{17}$, Frauke Zipp ${ }^{21}$, Iraide Alloza ${ }^{1,3}$, Manuel Comabella ${ }^{9}$, Christina M. Lill ${ }^{4,21,22,23}$ and Koen Vandenbroeck $1,3,24, *$ (D)

1 Neurogenomiks Laboratory, University of the Basque Country (UPV/EHU), 48940 Leioa, Spain; paloma.gomez.fernandez@gmail.com (P.G.-F.); aitzkoa.lopez_de_lapuente_portilla@med.lu.se (A.L.d.L.P.); nireaspe@hotmail.com (I.A.); jorgemena65@outlook.com (J.M.); a.urtasun.arricaberri@gmail.com (A.U.); iraide.alloza@gmail.com (I.A.)

2 Department of Laboratory Medicine, Lund University, SE-221 00 Lund, Sweden

3 Inflammation \& Biomarkers Group, Biocruces Bizkaia Health Research Institute, 48903 Barakaldo, Spain

4 Genetic and Molecular Epidemiology Group, Lübeck Platform for Genome Analytics, Institutes of Neurogenetics and Cardiogenetics, University of Lübeck, 23552 Lübeck, Germany; vivian.altmann@uni-luebeck.de (V.A.); christina.lill@uni-luebeck.de (C.M.L.)

5 Department of Cell Biology and Immunology, Instituto de Parasitología y Biomedicina López Neyra (IPBLN), CSIC, 18002 Granada, Spain; lindo@ipb.csic.es

6 Multiple Sclerosis Group, Biodonostia Research Institute, Paseo Doctor Begiristain, s/n, 20014 San Sebastián, Spain; David.otaegui@biodonostia.org (D.O.); tamara.castillo@biodonostia.org (T.C.-T.)

7 Instituto de Investigación Sanitaria del Hospital Clínico San Carlos, IdISSC, 28014 Madrid, Spain; elena.urcelay@salud.madrid.org (E.U.); lauraep80@yahoo.es (L.E.-P.)

8 Department of Neurology, Cruces Hospital, S/N, 48903 Barakaldo, Spain; alfredo.r.antiguedad.z@gmail.com

9 Servei de Neurologia-Neuroimmunologia, Centre d'Esclerosi Múltiple de Catalunya (Cemcat), Institut de Recerca Vall d'Hebron (VHIR), Hospital Universitari Vall d'Hebron, Universitat Autònoma de Barcelona, 08007 Barcelona, Spain; sunnymalhotra4u24@gmail.com (S.M.); xavier.montalban@cem-cat.org (X.M.); manuel.comabella@vhir.org (M.C.)

10 Department of Neurology, Medical Faculty, Heinrich-Heine University Düsseldorf, 40225 Düsseldorf, Germany; Orhan.Aktas@uni-duesseldorf.de

11 Department of Neurology, University of Wuerzburg, 97080 Wuerzburg, Germany; Mathias.Buttmann@ckbm.de

12 Department of Neurology, Caritas Hospital, 97980 Bad Mergentheim, Germany

13 Department of Neurology, Inselspital Bern, Bern University Hospital, University of Bern, 3011 Bern, Switzerland; andrew.chan@insel.ch

14 INSERM, Sorbonne University, Assistance Publique-Hopitaux de Paris (AP-HP), UMR 974 and Neuro-Myology Service, University Hospital Pitié-Salpêtrière, 75013 Paris, France; bertrand.fontaine@sorbonne-universite.fr

15 Nantes Université, CHU, INSERM, Centre de Recherche en Transplantation et Immunologie, UMR 1064, ATIP-Avenir, Equipe 5, 44093 Nantes, France; pierre-antoine.gourraud@univ-nantes.fr

16 CHU de Nantes, INSERM, CIC 1413, Pôle Hospitalo-Universitaire 11: Santé Publique, Clinique des données, 44000 Nantes, France

17 Department of Neurology, Neuroimmunological Section, University of Rostock, 18147 Rostock, Germany; michael.hecker@rocketmail.com (M.H.); uwe.zettl@med.uni-rostock.de (U.K.Z.)

18 Department of Human Genetics, Ruhr-University Bochum, 44801 Bochum, Germany; sabine.hoffjan@rub.de 
19 Institute of Human Genetics, University Medical Center Hamburg-Eppendorf, 20246 Hamburg, Germany; c.kubisch@uke.de

20 Institute of Clinical Neuroimmunology, Ludwig-Maximilians University, 80333 Munich, Germany; tania.kuempfel@med.uni-muenchen.de

21 Department of Neurology, Focus Program Translational Neuroscience, University Medical Center of the Johannes Gutenberg University Mainz, 55116 Mainz, Germany; luessi@uni-mainz.de (F.L.); frauke.zipp@unimedizin-mainz.de (F.Z.)

22 Section for Translational Surgical Oncology and Biobanking, Department of Surgery, University of Lübeck and University Medical Center Schleswig-Holstein, Campus Lübeck, 23552 Lübeck, Germany

23 Ageing Epidemiology Research Unit, School of Public Health, Imperial College, London SW71, UK

24 Ikerbasque, Basque Foundation for Science, 48013 Bilbao, Spain

* Correspondence: k.vandenbroeck@ikerbasque.org; Tel.: +34-946182622 (ext. 844748)

+ These authors contributed equally to this paper.

Received: 10 December 2019; Accepted: 3 January 2020; Published: 10 January 2020

\begin{abstract}
The IL22RA2 locus is associated with risk for multiple sclerosis (MS) but causative variants are yet to be determined. In a single nucleotide polymorphism (SNP) screen of this locus in a Basque population, rs28385692, a rare coding variant substituting Leu for Pro at position 16 emerged significantly $(p=0.02)$. This variant is located in the signal peptide (SP) shared by the three secreted protein isoforms produced by IL22RA2 (IL-22 binding protein-1(IL-22BPi1), IL-22BPi2 and IL-22BPi3). Genotyping was extended to a Europe-wide case-control dataset and yielded high significance in the full dataset $\left(p=3.17 \times 10^{-4}\right)$. Importantly, logistic regression analyses conditioning on the main known MS-associated SNP at this locus, rs17066096, revealed that this association was independent from the primary association signal in the full case-control dataset. In silico analysis predicted both disruption of the alpha helix of the H-region of the SP and decreased hydrophobicity of this region, ultimately affecting the SP cleavage site. We tested the effect of the p.Leu16Pro variant on the secretion of IL-22BPi1, IL-22BPi2 and IL-22BPi3 and observed that the Pro16 risk allele significantly lowers secretion levels of each of the isoforms to around $50 \%-60 \%$ in comparison to the Leu 16 reference allele. Thus, our study suggests that genetically coded decreased levels of IL-22BP isoforms are associated with augmented risk for MS.
\end{abstract}

Keywords: IL22RA2; IL-22 binding protein isoform; mutation; signal peptide; multiple sclerosis; autoimmune

\title{
1. Introduction
}

Multiple sclerosis (MS) is a chronic inflammatory CNS disorder triggered by environmental factors in individuals with a susceptible genetic background. Current research implies more than 230 autosomal risk variants, many of which are located within or close to genes exerting functions in the peripheral immune system or in CNS-resident microglia [1]. Single nucleotide polymorphisms (SNPs) located at the interleukin-22 receptor subunit alpha-2 (IL22RA2) gene locus were originally reported to be associated with risk for MS in Scandinavian [2] and Basque [3] populations. A series of genome-wide association studies (GWAS)-based approaches have subsequently firmly established the association of IL22RA2 with risk for MS [1,4-6].

The main known function of IL22RA2 is to produce interleukin-22 binding protein (IL-22BP), a secreted inhibitor of IL-22. IL-22, a member of the IL-10 family, is produced by a wide range of immune cells and can exert both pro- and anti-inflammatory effects $[7,8]$. Various lines of evidence suggest that the IL-22/IL-22BP axis has an important function in MS and neuroinflammation. Il22ra2-deficient mice experience a more benign course of disease in the experimental autoimmune encephalomyelitis (EAE) model [9]. The IL-22 receptor is highly expressed in blood-brain barrier (BBB) endothelial cells from 
patients with MS but not in healthy controls, and IL-22 contributes to the permeabilization of the BBB and recruitment of $\mathrm{CD}^{+} \mathrm{T}$ lymphocytes to the CNS [10]. A decrease of IL-22 levels correlates with the recovery phase of EAE in rats [11], and serum levels of IL-22 were found to be elevated in MS patients compared to healthy controls [12]. Perriard et al. [13] demonstrated that IL-22 targets astrocytes in the human brain and confers increased survival to these cells. They also found higher expression of IL-22BP mRNA in monocytes and monocyte-derived dendritic cells of MS patients compared to healthy controls.

IL22RA2 is capable of expressing three partially distinct isoforms that share an identical signal peptide (SP) at their N-terminus and lack intracellular and transmembrane domains but differ in their binding capacity of IL-22. Isoform 2 (UniProt nomenclature) shows the highest capacity of binding and inhibiting IL-22 [14,15], with a 20- to 1000-fold higher affinity than a soluble variant of the signal-transducing cell surface receptor [16-18]. Isoform 3 has also been demonstrated to bind IL-22, although with a similar affinity to that of the cell surface receptor $[16,19]$. Recently, we showed that the longest isoform, i.e., isoform 1, is not capable of binding IL-22 and displays hallmarks of a poorly secreted, intracellularly retained protein with intrinsic capacity to trigger the unfolded protein response (UPR; [20]).

Although the association of IL22RA2 with MS is now established and accumulating evidence points to an influence of IL-22 and IL-22RA2 in EAE and MS, the mechanism underlying the genetic association remains elusive. Here, we performed a SNP screen of the IL22RA2 locus in a Basque population in order to localize the most important association signal(s) within this locus and confirmed association of an infrequent coding SNP in a European cohort. We used dedicated in silico and wet experimentation methods to discover potentially causal variants that may explain the association of this gene with MS.

\section{Materials and Methods}

\subsection{Patients and Controls}

All patients were diagnosed with definite MS [21,22]. Written informed consent was obtained from all subjects, and the study was approved by the local ethics committees. Table 1 shows the clinical and demographic data of the patients and controls enrolled in this study. The fine-mapping was completed in the Bilbao dataset, comprising patients registered at the Basurto hospital (Bilbao, Basque Country, Spain) and controls provided by the Basque BioBank for Research-OEHUN (www.biobancovasco.org). Additionally, genotyping data of three SNPs (rs276466, rs10484798 and rs6570136) in the Bilbao cohort were available from the aforementioned screening [3], and these were included in the haplotype and logistic regression analyses.

The non-synonymous SNP, rs28385692, was further genotyped in additional patients and age-, gender- and ethnicity-matched controls from Donostia (Instituto Biodonostia, Basque Country, Spain), Barcelona (Hospital Vall d'Hebron), Madrid (Hospital Clínico S. Carlos), Andalucía (Instituto de Parasitología y Biomedicina "Lopez-Neyra"), Germany (various centers) and France (various centers) (Table 1). Considering the SNP with the lowest MAF (rs28385692, 1000G European frequency $=0.032$ ) and combining all datasets, this study had over $80 \%$ statistical power to detect allelic odds ratios $\geq 1.18$ with a type-1-error rate of alpha $=0.05$ (calculated with the Genetic Power Calculator allelic test [23]). When considering only the Bilbao dataset, we had over $80 \%$ power to detect odds ratios $\geq$ 1.8 , assuming a MAF $=0.032$ and a type-1-error of alpha $=0.05$.

The lead SNP from the 2011 GWAS [6], rs17066096, was also genotyped in all the above-mentioned validation cohorts, excluding France (Table 1). Additionally, data on the most significantly associated SNP in the fine mapping, rs202573, were available from Andalucía, Barcelona and Madrid from our previous study [3], and this SNP was newly genotyped in the Donostia and Germany cohorts. 
Table 1. Clinical and demographic features of patients and controls included in the genetic study. ${ }^{1}$ SD: standard deviation. ${ }^{2}$ RR: relapsing remitting MS. ${ }^{3}$ ScP: secondary progressive MS. ${ }^{4}$ PP: primary progressive MS. ${ }^{5}$ ND: not determined. ${ }^{6}$ EDSS: expanded disability status scale.

\begin{tabular}{|c|c|c|c|c|c|c|}
\hline \multicolumn{2}{|c|}{ Population } & \multirow{2}{*}{\begin{tabular}{|c|}
$\begin{array}{c}\text { Number } \\
\text { (\% Female) }\end{array}$ \\
$647(72.3)$
\end{tabular}} & \multirow{2}{*}{$\begin{array}{c}\begin{array}{c}\text { Age, Average } \\
\pm \text { SD }^{1}\end{array} \\
42.5 \pm 12.01\end{array}$} & \multirow{2}{*}{$\begin{array}{c}\mathbf{R R}^{2} \text { \& ScP }{ }^{3} / \\
\text { PP }^{4} / \text { Other/ND } \\
\end{array}$} & \multirow{2}{*}{$\begin{array}{c}\begin{array}{c}\text { Age at Onset, } \\
\text { Average } \pm \text { SD }\end{array} \\
30.42 \pm 10.17\end{array}$} & \multirow{2}{*}{$\begin{array}{c}\text { EDSS }^{6}, \\
\text { Mean } \pm \text { SD } \\
2.9 \pm 2.3\end{array}$} \\
\hline Bilho & Cases & & & & & \\
\hline Bilbao & Controls & $573(60.3)$ & $44.2 \pm 9$ & - & - & - \\
\hline \multirow{2}{*}{ Donostia } & Cases & $572(64.8)$ & $46.4 \pm 4.8$ & $84.8 / 3.8 / 4.8 / 6.6$ & $33.01 \pm 11.05$ & $2.79 \pm 2.7$ \\
\hline & Controls & $250(66)$ & $50.52 \pm 13.26$ & - & - & - \\
\hline \multirow{2}{*}{ Barcelona } & Cases & $676(63.3)$ & $40.17 \pm 12.93$ & $81.5 / 14.8 / 3.7$ & $31.6 \pm 9.9$ & $3.91 \pm 2.5$ \\
\hline & Controls & $910(52.7)$ & $40.2 \pm 12.9$ & - & - & - \\
\hline \multirow{2}{*}{ Madrid } & Cases & 899 (63.7) & $44.8 \pm 10.55$ & $79.7 / 6.9 / 4.7 / 8.7$ & $29.8 \pm 8.65$ & $2.56 \pm 2.13$ \\
\hline & Controls & $697(55.1)$ & $40.96 \pm 16.71$ & - & - & - \\
\hline \multirow{2}{*}{ Andalucía } & Cases & $1474(61)$ & $43 \pm 12$ & $47.4 / 1 / 9 / 42.6$ & $28.87 \pm 10.25$ & ND \\
\hline & Controls & 1777 (64.4) & $40.22 \pm 12.9$ & - & - & - \\
\hline \multirow{2}{*}{ Germany } & Cases & $3762(70.2)$ & $42.2 \pm 13.6$ & ND & ND & ND \\
\hline & Controls & $2972(60.1)$ & $41.1 \pm 14.05$ & - & - & - \\
\hline \multirow{2}{*}{ France } & Cases & $1344(63.6)$ & $44.3 \pm 11.8$ & ND & ND & ND \\
\hline & Controls & $768(60.4)$ & $39.6 \pm 13$ & - & - & - \\
\hline
\end{tabular}

\subsection{Selection and Genotyping of SNPS}

Ten haplotype-tagging SNPs were selected based on genotype data from the CEU + TSI population (HapMap release \#27) using an $r^{2}$ cut-off $=0.9$ and a MAF $=0.1$ (with the Multimarker Tagger Algorithm available on the HapMap website, www.hapmap.org, now defunct). In addition, SNP rs13217897 was selected based on its association with MS as reported by an independent group [2], and rs28385692 was included because of its potential functional effect, given its location in the coding region. Haplotype-tagging SNPs in the Bilbao cohort and rs28385692 in the Donostia cohort were genotyped using the iPLEX Sequenom MassARRAY platform in the Spanish National Genotyping Center (CEGEN, Santiago de Compostela, Spain, www.cegen.es). Genotyping of rs17066096, rs202573 and rs28385692 in the validation cohorts was performed using Taqman ${ }^{\circledR}$ Genotyping Assays, following the manufacturer's instructions. The genotyping efficiency was above $95 \%$ in all datasets, and all SNPs were in Hardy-Weinberg equilibrium in controls in each dataset.

\subsection{Statistical Analysis}

The statistical analysis was performed using PLINK v1.07 (http://zzz.bwh.harvard.edu/plink/) [24]. For the association analysis, a logistic regression with an additive model, adjusted for sex, was applied. The independence of association signals was assessed using a conditional logistic regression analysis. A chi-squared test was used to check for the Hardy-Weinberg equilibrium. A haplotype analysis was performed using Haploview v.4.2 (https://www.broadinstitute.org/haploview/haploview) [25]. Only the samples that had genotyping data available for all the SNPs were used for the haplotype calculation (for the Bilbao cohort, 375 cases and 441 controls; for the joint datasets, 6545 cases and 5713 controls). Haplotype blocks were defined by the confidence interval method [26]. Permutations were applied ( $n$ permutations $=1000)$ to correct for multiple comparisons in the haplotype analysis. Statistical power was calculated using the CaTS power calculator at www.sph.umich.edu/csg/abecasis/CaTS/ [27]. Secretion levels of Leu16 IL-22BP isoforms compared to those of Pro16 variants were compared used Student's unpaired $t$-test. 


\subsection{Functional Annotation of SNPS}

Each of the associated SNPs and their proxies $\left(r^{2} \geq 0.8\right.$ in European populations of the 1000 Genomes pilot project as retrieved from the SNAP Proxy Search tool (https://www.broadinstitute. org/mpg/snap/) [28] were functionally annotated using the Ensembl Variant Effect Predictor (VEP) (https://www.ensembl.org/info/docs/tools/vep/index.html) [29] and the RegulomeDB (http://www. regulomedb.org/) [30].

\subsection{In silico Analysis of the Effect of the Leu to Pro Transition Coded by rs28385692 on Signal Peptide Characteristics}

PredictSNP web server (https://loschmidt.chemi.muni.cz/predictsnp1/) [31], Meta-SNP (http://snps.biofold.org/meta-snp/) [32] and Ensembl VEP tool (http://www.ensembl.org/Homo_ sapiens/Variation/Mappings? $\mathrm{db}=\mathrm{core} ; \mathrm{r}=6: 137161203-137162203 ; \mathrm{v}=\mathrm{rs} 28385692 ; \mathrm{vdb}=\mathrm{variation} ; \mathrm{vf}=$ 104559210\#ENST00000296980_104559210_G_tablePanel) [29,33] were used to estimate the functional effect of p.Leu16Pro mutation on IL-22BP. The SignalP 3.0 [34], Phobius [35], PsiPred [36], SignalP 5.0 (http://www.cbs.dtu.dk/services/SignalP/) [37], PrediSi [38], and Signal-3L tools were used to predict the effects of the p.Leu16Pro substitution on SP function in IL-22BP. Secondary structure features of the wildtype/mutant proteins were predicted by PsiPred [39], RaptorX (http://raptorx.uchicago.edu/) [40] and SABLE [41].

\section{6. p.Leu16Pro Mutagenesis of the Three IL-22BP Isoforms}

The expression plasmid for IL22RA2v1 was constructed as described in our previous work [20], IL22RA2v2 and IL22RA2v3 expression plasmids were purchased from OriGene Technologies (RC219095, Rockville, MD, USA) and GenScript (Ohu00490, Piscataway, NJ, USA), respectively. The p.Leu16Pro mutants of IL-22BPi1, 2 and 3 were generated using the GENEART ${ }^{\circledR}$ site-directed mutagenesis system (A13282, Invitrogen, Waltham, MA, USA) from the IL22RA2v1, 2 and 3 expression plasmids following the manufacturer's instructions. The site-directed mutagenesis primer design was also done following the manufacturer's instructions. Briefly, both primers contained the desired mutation centrally located and were $100 \%$ complementary with no overhangs, and with lengths between 30 and 45 nucleotides. The designed primers, purchased from IDT, were purified by HPLC to increase mutagenesis efficiency. PCR was performed using a Verity thermocycler (Applied Biosystems, Waltham, MA, USA) with the following primers: IL22RA2_p.Leu16Pro_FW: 5'-TCATCAGTTTCTTCCCTACTGGTGTAGCAGG-3' and IL22RA2_p.Leu16Pro _RV: 5'-CCTGCTACACCAGTAGGGAAGAAACTGATGA-3'. The PCR conditions used were: 1 cycle at $37^{\circ} \mathrm{C}$ for $20 \mathrm{~min}$ and $94{ }^{\circ} \mathrm{C}$ for $45 \mathrm{~s}, 18$ cycles at $94{ }^{\circ} \mathrm{C}$ for $45 \mathrm{~s}$, $57{ }^{\circ} \mathrm{C}$ for $45 \mathrm{~s}$ and $72{ }^{\circ} \mathrm{C}$ for $6 \mathrm{~min}, 1 \mathrm{cycle}$ at $72{ }^{\circ} \mathrm{C}$ for $10 \mathrm{~min}$ and a final holding stage at $4{ }^{\circ} \mathrm{C}$. The resulting constructs were sequenced to confirm point mutations of IL22RA2 variant constructs. The p.Leu16Pro mutant plasmids were transformed into DH5 $\alpha$-T1R E. coli competent cells following the manufacturer's instructions. Plasmids were purified with EndoFree Plasmid Kit (Qiagen, Hilden, Germany), quantified by a NanoDrop Spectrophotometer (ThermoFisher Scientific, Waltham, MA, USA) and visually assessed via agarose gel electrophoresis.

\subsection{Assessment of Effect of p.Leu16Pro Variant on Secretion of IL-22BP Isoforms}

HEK293 cells were cultured in Dulbecco's Modified Eagle Medium (DMEM: D5796, Sigma, St. Louis, MO, USA) supplemented with $10 \%$ fetal bovine serum (FBS; F9665, Sigma, St. Louis, $\mathrm{MO}, \mathrm{USA}$ ) at $37^{\circ} \mathrm{C}$ and $5 \% \mathrm{CO}_{2}$ in a humidified incubator. Cells were seeded on a 24-well plate at $500 \mu \mathrm{L} /$ well at a density of $3 \times 10^{4}$ cells/well. Cells were transfected with the indicated expression plasmids when they reached $60 \%-70 \%$ confluency using MACSfectin Reagent (130-098-412, Miltenyi, Bergisch Gladbach, Germany). Twenty-four hours after transfection, conditioned media were collected and cells washed three times with cold PBS prior cell lysis in RIPA buffer ( $25 \mathrm{mM}$ Tris- $\mathrm{HCl}, 150 \mathrm{mM}$ $\mathrm{NaCl}, \mathrm{pH} 7.6$; plus $1 \% \mathrm{~N}-40,1 \%$ sodium deoxycholate, and $0.1 \% \mathrm{SDS}$ ) in the presence of protease 
inhibitors (11697498001, Roche, Basel, Switzerland). All cell lysates were quantified for total protein using BCA kit (23225, ThermoFisher Scientific); equal amounts of total protein were resuspended in reducing loading buffer and resolved on SDS-PAGE for further immunoblotting. For acetone precipitation of conditioned media, $4 \mathrm{~h}$ prior media collection, cells were carefully washed five times with pre-warmed serum-free medium (SFM; 12-764Q, Lonza, Basel, Switzerland) to remove serum proteins and SFM supplemented with L-glutamine (G5792, Sigma) was added for a further $4 \mathrm{~h}$. Conditioned SFM were acetone precipitated with four volumes of ice-cold acetone and incubated on ice for $10 \mathrm{~min}$ followed by centrifugation at $21,000 \times \mathrm{g}$ a $4{ }^{\circ} \mathrm{C}$. Pellets were resuspended in reducing loading buffer and resolved on SDS-PAGE for further immunoblotting. The antibodies used in this study are the following: anti-FLAG (1:1000; 2043-1-AP, Proteintech, Rosemont, IL, USA); anti-IL-22BP (1:1000; AF1087 and BAF1087, R\&D Systems, Minneapolis, MN, USA); anti-IL-22BP (1:1000; ab133965, Abcam, San Francisco, CA, USA); anti-tubulin (1:1000; A01490, GenScript, Piscataway, NJ, USA) and all HRP-conjugated secondary antibodies were purchased from Jackson ImmunoResearch. IL-22BP ELISA capable of detecting the three isoforms was performed as previously described [20].

\subsection{Replacement of the SP of IL-22BPi2 with the IL17A SP}

The IL17SP_IL22RA2v2 construct was generated from two overlapping fragments; the first one containing the terminal SgfI restriction site followed by the sequence of the SP of IL-17A and the beginning of IL-22BPi2 mature protein, and the second one, containing the end of IL-17A SP followed by IL-22BPi2 mature protein flanked by Mlu restriction site. The overlapping fragment was amplified and digested with $S g f I$ and MluI restriction enzymes and cloned into pCMV6-Entry vector. Primers for amplification of the IL17A_Signal peptide fragment were: FW: 5'-GCCGCGATCGCCATGACTCCTGGGAAGACC-3' and RV:5'-AGGCTTCAGAGACTCATGCG TTGACTGAGTGATTGTGATTCCTGCCTTCACTATGGCCTCCAGGCTC-3' . Primers for amplification of the IL22RA2v2 mature fragment were: FW $5^{\prime}$-GAGCCTGGAGGCCATAGTGAAGGCAGGAATCA CAATCACTCAGTCAACGCATGAGTC TCTG-3' and RV: 5'-CGTACGCGTTGGAATTTCCACACA TCTCTC-3'.

\subsection{Flow Cytometry Analysis}

HEK293 cells were transfected with expression vectors for wild-type or p.Leu16Pro mutant IL-22BPi2, collected after $24 \mathrm{~h}$ and washed with flow cytometry buffer (FC Buffer; PBS, 0.5\% BSA and $2 \mathrm{mM}$ EDTA, $\mathrm{pH}$ 7.2). Single cell suspensions were fixed with $4 \%$ paraformaldehyde for $10 \mathrm{~min}$ at room temperature followed by permeabilization with $90 \%$ of ice-cold methanol for $30 \mathrm{~min}$ at $4{ }^{\circ} \mathrm{C}$. Cells were blocked with $1 \%$ BSA for 15 min at RT, incubated with anti-IL-22BP primary antibody $(1: 250 ; 66190$, Proteintech) for $30 \mathrm{~min}$ at room temperature and followed by another 30-minute incubation period with anti-mouse-FITC conjugated secondary antibody (1:500; AMI4608, ThermoFisher) protected from light. Two washes were performed after each step with FC buffer. Immunostained cells were analyzed using a MACSQuant Analyzer (Miltenyi).

\section{Results}

\subsection{The p.Leu16Pro coding SNP rs28385692 is Associated with Risk for Multiple Sclerosis}

A total of 15 SNPs in a $~ 100-k b$ interval around IL22RA2 were analyzed in the Bilbao cohort. Of these, five showed nominally significant $(p<0.05)$ association with MS (Figure 1, Table 2). The most strongly associated SNP was rs202573 (OR $=1.27, p=0.007)$, which had already been the index SNP in the initial screening [3]. This intronic SNP is in weak LD in the Bilbao dataset $\left(r^{2}=0.06, \mathrm{D}^{\prime}=0.31\right)$ with the most significant GWAS-derived top SNP found at the IL22RA2 locus, i.e., rs17066096 [1,4-6]. Rs17066096 showed no association in the Bilbao dataset. Logistic regression analysis of all SNPs conditioning on rs202573 did not reveal any other independent SNP signal reaching statistical significance including rs17066096, suggesting that the main effect observed in this population is driven 
by rs202573 (Appendix A Table A1). Haplotype analysis did not show any haplotype displaying a higher significance than single SNPs in the IL22RA2 region (Table A2).

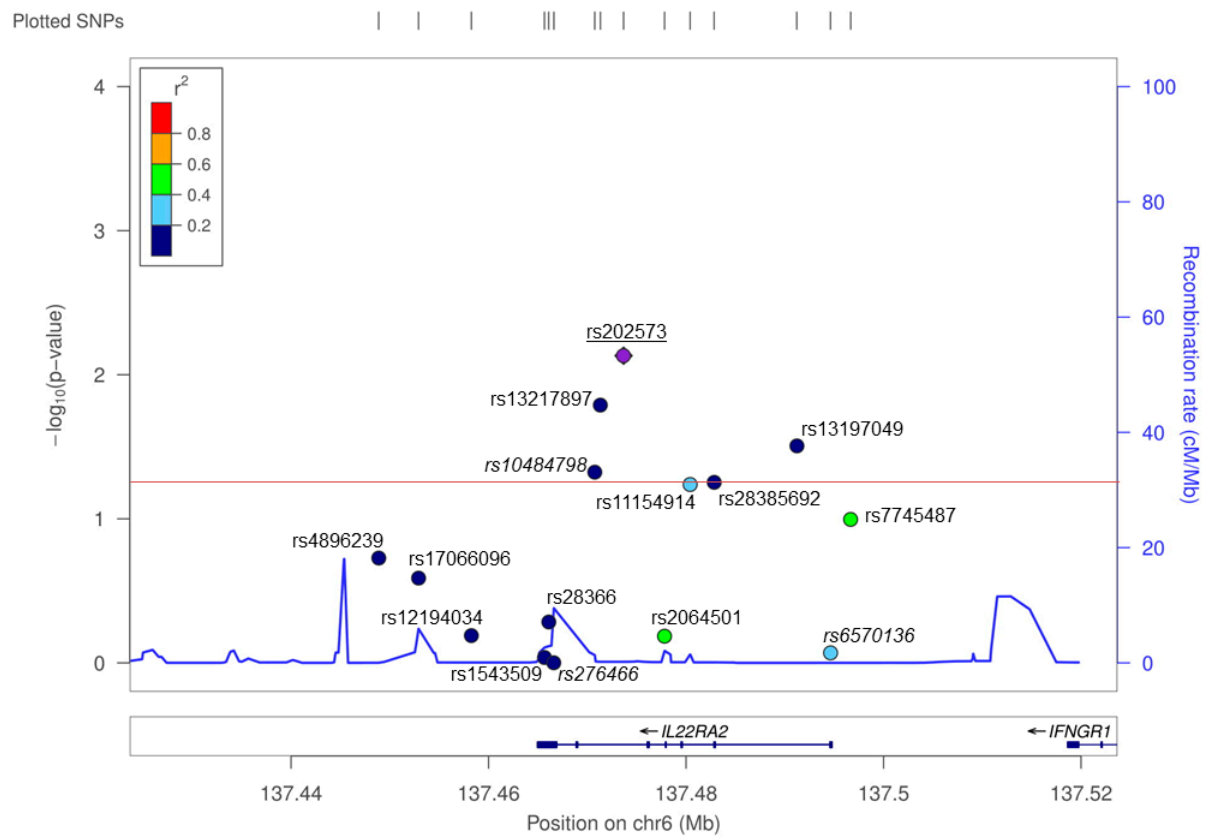

Figure 1. A single nucleotide polymorphism (SNP) screen of the IL22RA2 locus in the Bilbao dataset. SNPs, depicted with dots in different colors depending on $r^{2}$ values with respect to the index SNP rs202573, are plotted as a function of their log-converted $p$-value (left $\mathrm{Y}$ axis) and their position on chromosome 6 according to hg19 assembly of the human genome ( $\mathrm{X}$ axis). The recombination rate across the locus is provided on the right $\mathrm{Y}$ axis. The red line represents the significance threshold $(p=0.05)$. SNPs genotyped in the primary screening [3] are shown in italics. rs202573, which was genotyped both in the previous and in the present study, is underlined.

Table 2. Association values of SNPs included in the mapping analysis in the Bilbao dataset. ${ }^{1}$ Position is according to the hg19 genome build. ${ }^{2}$ RAF: risk allele frequency. ${ }^{3}$ OR: odds ratio. ${ }^{4} \mathrm{CI}$ : confidence interval.

\begin{tabular}{cccccccc}
\hline SNP & Position $^{\mathbf{1}}$ & Risk Allele & RAF $^{\mathbf{2}}$ Cases & RAF Controls & Other Allele & $p$ & OR $^{\mathbf{3}} \mathbf{( 9 5}^{\mathbf{9}} \mathbf{C I}^{\mathbf{4}} \mathbf{)}$ \\
\hline rs4896239 & $137,448,873$ & $\mathrm{C}$ & 0.52 & 0.50 & $\mathrm{~T}$ & 0.19 & $1.116(0.942-1.31)$ \\
rs17066096 & $137,452,908$ & $\mathrm{G}$ & 0.29 & 0.27 & $\mathrm{~A}$ & 0.26 & $1.132(0.92-1.34)$ \\
rs12194034 & $137,458,262$ & $\mathrm{~A}$ & 0.23 & 0.22 & $\mathrm{~T}$ & 0.65 & $1.047(0.86-1.274)$ \\
rs1543509 & $137,465,656$ & $\mathrm{C}$ & 0.15 & 0.14 & $\mathrm{~T}$ & 0.92 & $1.012(0.797-1.285)$ \\
rs28366 & $137,466,087$ & $\mathrm{C}$ & 0.24 & 0.23 & $\mathrm{~T}$ & 0.52 & $1.066(0.88-1.297)$ \\
rs276466 & $137,466,614$ & $\mathrm{~A}$ & 0.78 & 0.78 & $\mathrm{G}$ & 0.99 & $1.001(0.799-1.25)$ \\
rs10484798 & $137,470,756$ & $\mathrm{~A}$ & 0.76 & 0.72 & $\mathrm{G}$ & 0.05 & $1.23(1.0-1.508)$ \\
rs13217897 & $137,471,327$ & $\mathrm{G}$ & 0.83 & 0.79 & $\mathrm{~A}$ & 0.02 & $1.291(1.05-1.591)$ \\
rs202573 & $137,473,672$ & $\mathrm{~A}$ & 0.33 & 0.28 & $\mathrm{G}$ & 0.007 & $1.273(1.067-1.518)$ \\
rs2064501 & $137,477,823$ & $\mathrm{~T}$ & 0.50 & 0.49 & $\mathrm{C}$ & 0.65 & $1.039(0.879-1.226)$ \\
rs11154914 & $137,480,411$ & $\mathrm{G}$ & 0.19 & 0.16 & $\mathrm{~A}$ & 0.06 & $1.23(0.99-1.524)$ \\
rs28385692 & $137,482,840$ & $\mathrm{C}$ & 0.02 & 0.01 & $\mathrm{~T}$ & 0.05 & $1.972(0.983-3.954)$ \\
rs13197049 & $137,491,211$ & $\mathrm{~A}$ & 0.83 & 0.80 & $\mathrm{~T}$ & 0.03 & $1.260(1.021-1.556)$ \\
rs6570136 & $137,494,622$ & $\mathrm{~A}$ & 0.46 & 0.45 & $\mathrm{G}$ & 0.85 & $1.017(0.847-1.222)$ \\
rs7745487 & $137,496,672$ & $\mathrm{~A}$ & 0.18 & 0.15 & $\mathrm{G}$ & 0.10 & $1.201(0.96-1.496)$ \\
\hline
\end{tabular}

Interestingly, an infrequent non-synonymous SNP (rs28385692; changes Leu to Pro at position 16 of the SP shared by the three IL-22BP isoforms) was at the limit of statistical significance in the Bilbao dataset (Figure 1 and Table $2 ; p=0.05, \mathrm{OR}=1.972,95 \% \mathrm{CI}=0.983-3.954$ ). The risk allele of this SNP (C) is comparatively rare $(\mathrm{MAF}=0.015)$. Notably, haplotype analyses of all genotyped SNPs revealed that 
among all the haplotypes that were present with a frequency higher than $0.5 \%$ in the Bilbao cohort, the $C$ allele was present only in one haplotype, which contained the risk alleles of four additional SNPs that displayed significant associations in the Bilbao dataset (Figure A1). The association of rs28385692 with MS strengthened when adding 250 controls and 572 cases from nearby Donostia to our analysis, also located in the Basque Country ( $p=0.03, \mathrm{OR}=1.88,95 \% \mathrm{CI}=1.08-3.282)$. Next, we validated this finding in independent Spanish and European cohorts comprising a total of 8960 cases and 7613 controls. The joint analysis of the discovery and validation cohorts confirmed the association of rs 28385692 with MS $\left(p=3.6 \times 10^{-4}, \mathrm{OR}=1.262,95 \% \mathrm{CI}=1.11-1.434\right.$ (Figure 2a).

(a)

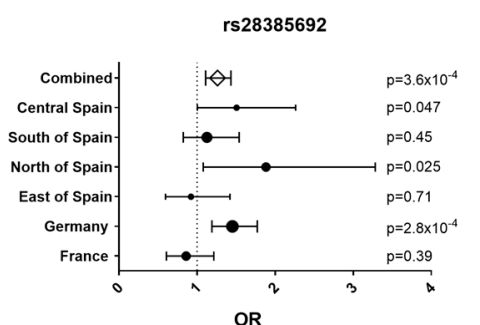

(b)

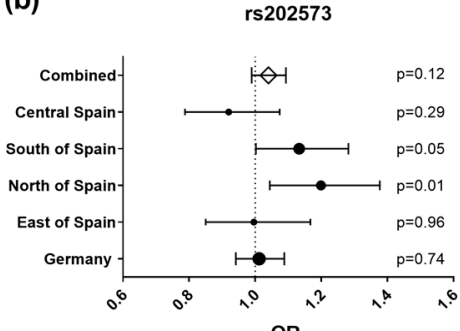

(c)

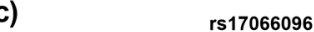

Figure 2. Forest plots representing effect size estimates (OR, 95\% confidence interval) of the risk alleles of rs28385692 (a), rs202573 (b) and rs17066096 (c) in the study populations: Central Spain (Madrid area), South of Spain (Andalucía), North of Spain (Basque Country), East of Spain (Barcelona area), Germany, and France, and in the combined dataset. The dots' size is proportional to the sample size of each population.

To further delineate the findings of this mapping effort, our original index SNP, rs202573, and the GWAS-derived top SNP, rs17066096 [4,6], were also genotyped in all validation datasets except France. Upon combining all available datasets, we found a significant association of rs17066096 $\left(p=9.9 \times 10^{-5}\right.$, $\mathrm{OR}=1.11,95 \% \mathrm{CI}=1.054-1.172)$, but not rs202573 ( $p=0.12, \mathrm{OR}=1.04,95 \% \mathrm{CI}=0.99-1.09)$ (Figure 2b,c). Logistic regression analysis on the combined datasets revealed that the associations of rs17066096 and rs28385692 are statistically independent, since conditioning on one SNP did not abolish association of the other (Table 3). None of the haplotypes formed by these three SNPs increased the statistical evidence for association compared to the single SNPs in the combined datasets, but, as seen in the dataset from Bilbao, the $\mathrm{C}$ allele of the infrequent SNP rs28385692 appeared only in the same haplotype as the risk alleles of the other two SNPs (Figure A2).

Table 3. Association values of the three SNPs in the discovery + validation datasets conditioned on rs17066096 and rs28385692. ${ }^{1}$ OD: odds ratio. ${ }^{2}$ CI: confidence interval.

\begin{tabular}{cccccc}
\hline & & \multicolumn{2}{c}{ Conditioned to rs17066096 } & \multicolumn{2}{c}{ Conditioned to rs28385692 } \\
\hline SNP & Reference (minor) Allele & $p$ & OR $^{\mathbf{1}} \mathbf{( 9 5 \% \mathbf { C I } ^ { \mathbf { 2 } } )}$ & $\boldsymbol{p}$ & OR $\mathbf{( 9 5 \%}$ CI) \\
\hline rs17066096 & G & NA & NA & 0.001042 & $1.098(1.039-1.162)$ \\
rs202573 & A & 0.2424 & $1.029(0.981-1.079)$ & 0.3093 & $1.033(0.9702-1.1)$ \\
rs28385692 & C & 0.001146 & $1.098(1.101-1.476)$ & NA & NA \\
\hline
\end{tabular}

We next attempted to define which of the associated SNPs were most likely to have functional effects based on in silico predictions. To this purpose, we analyzed the five significant variants from the original Bilbao SNP screen and SNPs that were significantly associated in the validation effort, i.e., rs17066096 and rs28385692, using VEP and RegulomeDB (Table 4). As this study was based on a haplotype-tagging method, the observed association signals were considered to be representative of SNPs in linkage disequilibrium (LD) with the associated markers, and therefore, proxies for the associated SNPs were also included in the analysis. Neither rs202573, the most associated SNP in the Bilbao dataset, nor the only proxy of this SNP which met the $r^{2}$ threshold $\left(r^{2}=0.8\right)$ to be included in 
the analysis, were predicted to overlap with any regulatory feature. rs17066096 did not show evidence to be functional either, but two of its perfect proxies $\left(r^{2}=1\right)$, rs17066063 and rs62420820, displayed a moderate regulatory potential based on RegulomeDB. rs17066063 was the SNP with the highest regulatory potential based on concordance between RegulomeDB (score 3a) and VEP (consequence: TF binding site variant). rs 28385692 was predicted to lie within a regulatory region, although the level of evidence supporting this is modest (score 5 according to RegulomeDB).

Table 4. Functional predictions of associated SNPs and proxies. SNPs with significant associations in the mapping exercise or in the discovery + validation cohorts and their proxies $\left(r^{2}>0.8\right.$ in 1000 Genomes Phase III CEU population) were assessed using VEP and RegulomeDB. ${ }^{1}$ Minor allele frequency is based on European populations in the 1000 Genomes Project Phase III.

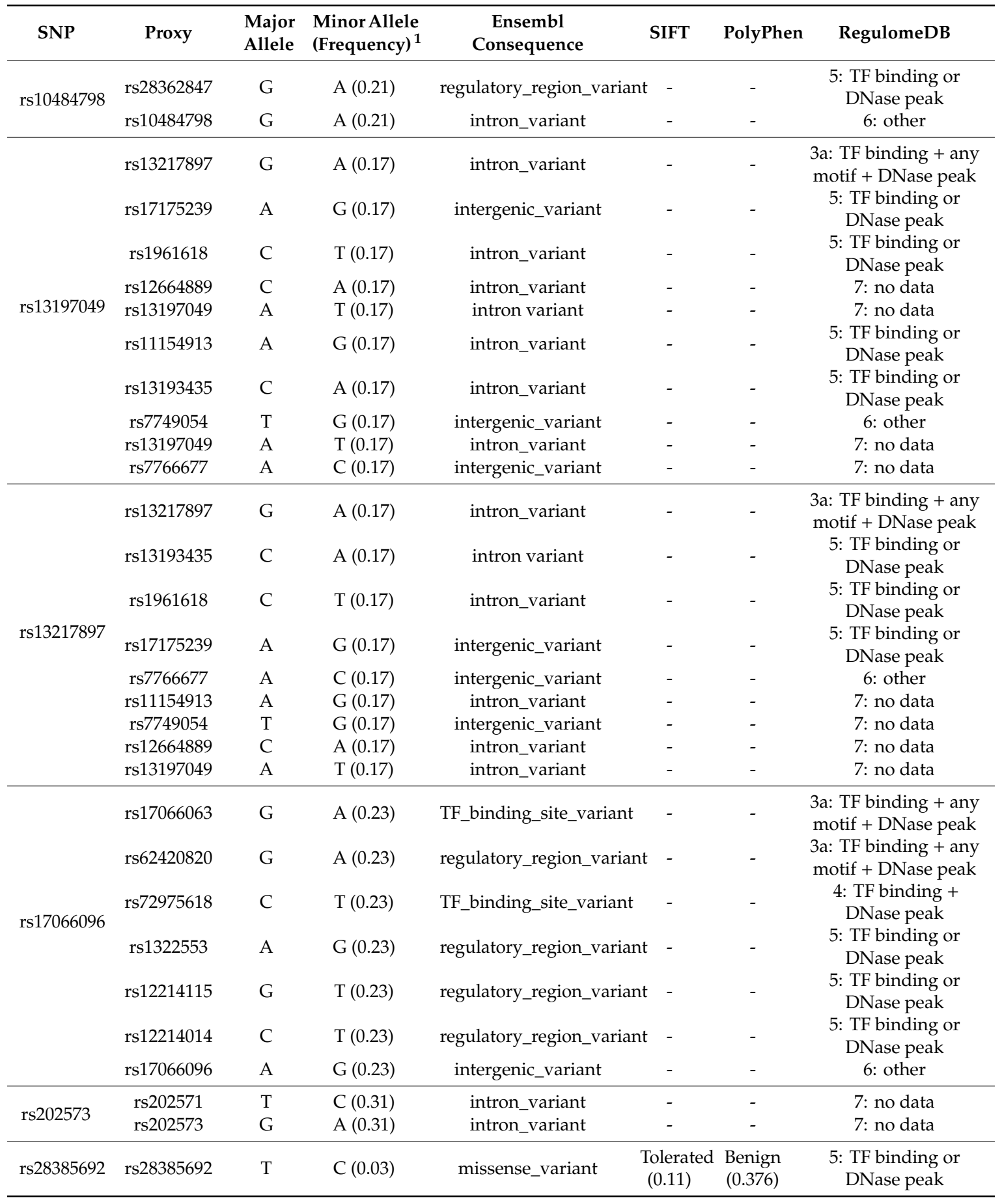




\subsection{In Silico Analysis of the p.Leu16Pro Variant}

We evaluated in silico whether rs 28385692 is functionally neutral or deleterious by using computational tools based on different approaches [42]; i.e., PredictSNP [31], Meta-SNP [32] and Ensembl VEP tool $[29,33]$. A summary of the features of each tool used in this study is represented in Table A3. PredictSNP indicated that the overall result of the p.Leu16Pro transition in the SP was deleterious for all three isoforms (Figure A3a). However, Meta-SNP [32] anticipated a neutral effect for the p.Leu16Pro point mutation in all IL-22BP isoforms (Figure A3b). Finally, the pathogenicity prediction tools included in Ensembl VEP tool predicted the substitution not to be deleterious except for a sub-analysis based on the Mutation Assessor tool available in Ensembl VEP, which attributed a medium level of functional impact to this variant (Figure A3c). Thus, the overall results obtained with the computational tools were not robust enough to unequivocally assign a functional effect to the p.Leu16Pro variant. As this variant occurs in the SP of IL-22BP protein, its potential effect on structural aspects pertinent to SP biological function per se was assessed in more detail by in silico methods. Coding SNPs in signal peptides may alter translocation efficiency, cleavage sites, as well as post-cleavage events [43]. We analyzed the charge, the hydrophobicity, and the helix-breaker amino acid residues comprised in the SP of IL-22PB, as well as the modifications introduced by the p.Leu16Pro variant (Figure 3). The Leu16 residue is one of three leucines that contribute to the IL-22BP SP hydrophobic core H-region, a stretch of hydrophobic amino acids with propensity to form a single alpha-helix. However, its replacement, Pro16, is a helix-breaker neutral residue with restricted conformational flexibility that has no free hydrogen to contribute to helix stability. Results obtained with various signal peptide cleavage site and secondary structure prediction tools are represented in Figure 3a. While SignalP-3.0 [34] and PSIPRED [36] did not predict any change in the cleavage site, Phobius [35] predicted the p.Leu16Pro polymorphism to shift the signal peptide cleavage site from the 21st to 20th residue. The three software applications coincided in predicting a shortening of the hydrophobic core of the signal peptide. Other prediction tools were applied as well and these predicted similar structural effects (Figure A4).

\subsection{The p.Leu16Pro Variant Decreases Secretion of IL-22BPi1, IL-22BPi2 and IL-22BPi3}

To experimentally verify the effect of this variant on secretion, we performed site-directed mutagenesis to change Leu16 to Pro in the signal peptide of the three wild-type IL-22BP isoforms cloned in expression vectors reported before [20]. HEK293 cells were individually transfected with these vectors, and both intracellular and secreted levels of IL-22BPi1, IL-22BPi2 and IL-22BPi3 were measured by ELISA. Compared to Leu16, Pro16 strongly decreased the secreted levels of each isoform (Figure 4a). This was mirrored by nonsignificant trends towards decreased intracellular levels of IL-22BPi1 and IL-22BPi2. Cell lysates as well as acetone precipitates of conditioned medium of HEK293 cells transfected to produce Leu16 or Pro16 forms of IL-22BPi1 and IL-22BPi2 were analyzed by immunoblot and this revealed decreased intracellular (51 kDa for IL-22BPi1 and $48 \mathrm{kDa}$ for IL-22BPi2) and secreted (56 kDa for IL-22BPi2) levels of the mutant forms (Figure $4 \mathrm{~b}$ ), while transfection efficiencies as measured by RT-qPCR through quantification of mRNA levels were similar (Figure 4c). These observations are compatible with the effects predicted by the above in silico analysis, suggesting that the Pro16 variant renders import of the precursor IL-22BP isoforms into the endoplasmic reticulum less efficient. Flow cytometry analysis also revealed a decrease of $41 \%$ to $33 \%$ in IL-22BP+ cells following transfection of HEK293 with Leu16 or Pro16 vectors encoding IL-22BPi2, respectively (Figure A5). We also assessed the efficiency of the native signal peptide of IL-22BPi2 in facilitating secretion by replacing it with that of IL-17, an efficiently secreted protein [20]. This modification resulted in much higher levels of both intracellular and secreted IL-22BPi2 (Figure 5), suggesting that the wild-type IL-22BP signal peptide is less efficiently engaged by the co-translational targeting and translocation machinery across the ER membrane than that of IL-17, which may affect overall IL-22BP biogenesis [44]. 
(a) SignalP 3.0

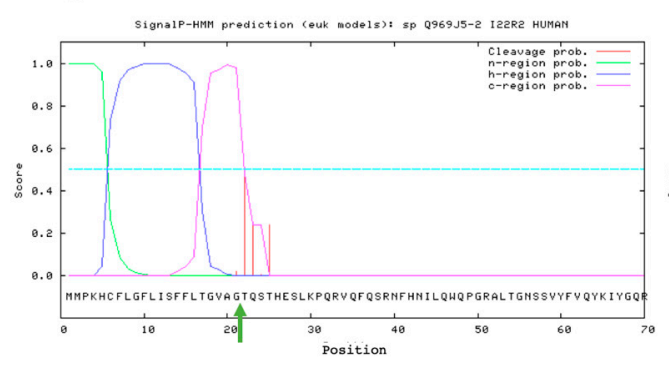

Phobius

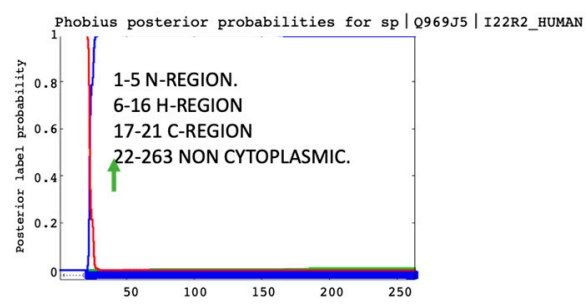

PsiPred

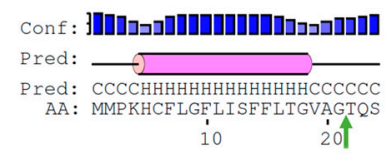

IL-22BP PI6
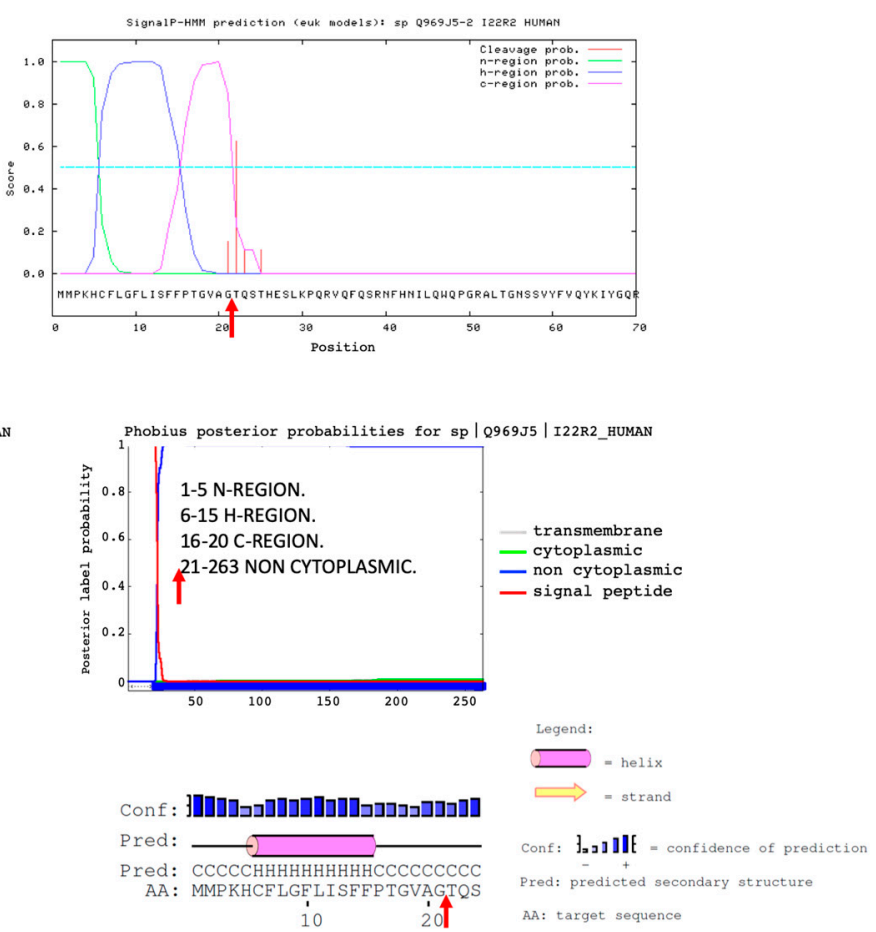

(b)

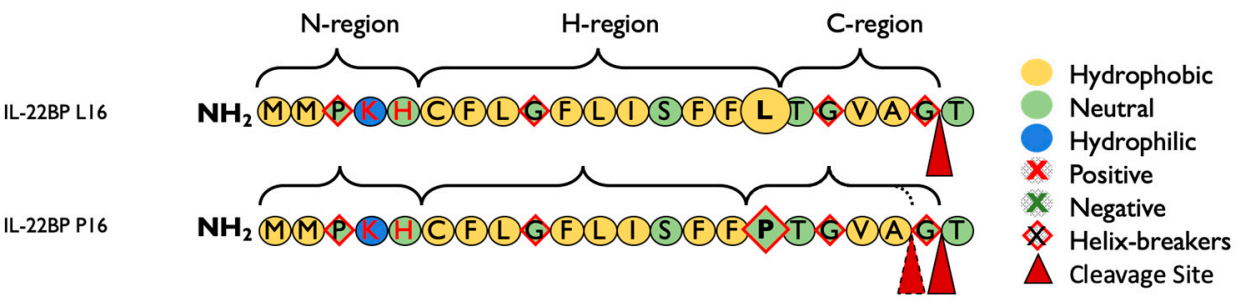

Figure 3. Prediction of the effect of the p.Leu16Pro amino acid change on the IL-22BP signal peptide structure and cleavage site of mature IL-22BP. (a) The signal peptide cleavage site indicated with green (wt, Leu16) or red (Pro16) arrows, was predicted using SignalP-3.0, Phobius and PsiPred software. The cleavage site for the canonical sequence is predicted to occur between positions 21 and 22 by the three software applications used. The p.Leu16Pro variant causes a shift in the predicted cleavage site to between position 20 and 21 according to Phobius but not SignalP 3.0 and PsiPred each of which predicted identical cleavage sites to the canonical sequence. All software coincided in predicting a decrease in the length of the H-region in the mutant form. (b) Representation of the composition, hydrophobicity and charge of the amino acids that comprise the signal peptide of IL-22BP. The three domain structures of the IL-22BP signal peptide are represented based on the overall results obtained in (a), and consist of the N-region, a hydrophilic positively charged N-terminal region; the H-region, a hydrophobic core region; and the C-region, a polar uncharged C-terminal region that is recognized by signal peptidase.

\section{Discussion}

In this study, we identified an infrequent functional variant (rs28385692) in IL22RA2, which confers risk to MS independently of the major GWAS-derived signal, and we were able to show that this variant has immediate functional effects by reducing secreted IL-22BP levels in transfected HEK293 cells. 
(a)
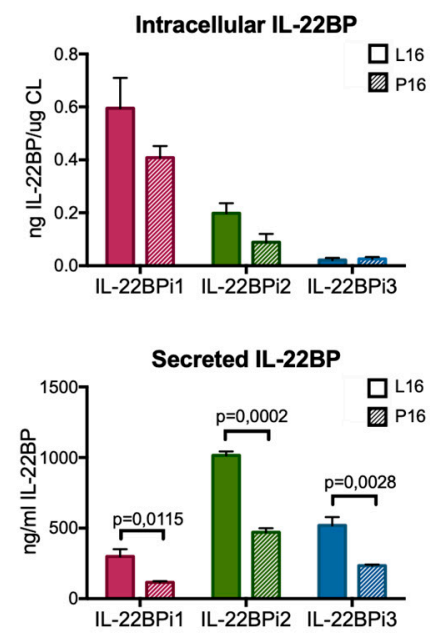

(b)

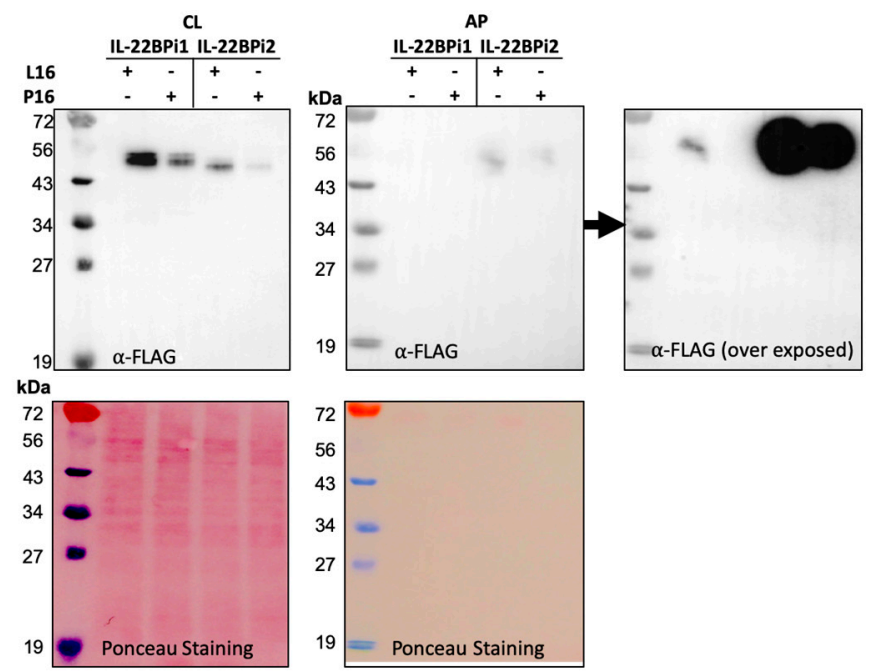

(c)
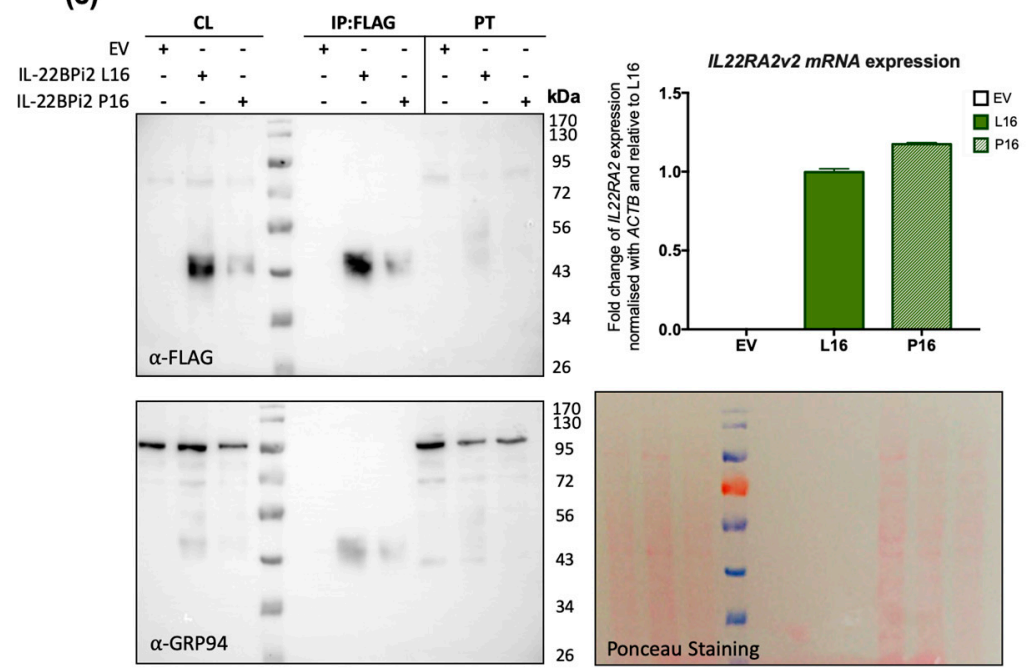

Figure 4. The Pro16 variant in the SP of the three IL-22BP isoforms is associated with decreased secretion levels compared to the Leu16 variant. (a) HEK293 cells were transfected with the indicated expression plasmids, $24 \mathrm{~h}$ later cells were lysed and the conditioned medium collected. Intracellular and secreted IL-22BP isoform protein levels were measured by ELISA (mean \pm SEM; $n=3$; $p$-values by unpaired $t$-test). (b) HEK293 cells were transfected with the indicated expression plasmids, $24 \mathrm{~h}$ later cells were lysed and the conditioned medium was subjected to acetone precipitation (AP). Both cell lysates (CL) and AP were resolved by SDS-PAGE under non-reducing conditions and immunoblotted against FLAG (Ponceau staining served as loading control). For AP, the immunoblot membrane was subjected to longer exposure times. (c) HEK293 cells were transiently transfected with the indicated expression vectors (EV denotes empty vector), $24 \mathrm{~h}$ later cells were lysed and RNA purified. Intracellular IL-22BP protein was immunoaffinity-purified with FLAG agaroses and detected by WB following FLAG purification and in cell lysates (CL) and pass through fraction (PT). GRP94 detection and Ponceau staining served as loading controls. Transfection efficiency was measured by IL22RA2 RT-qPCR relative to the housekeeping gene ACTB. Mean \pm SEM of three technical replicates. Note that as previously observed [20], immunoreactive bands corresponding to intracellular IL-22BP isoforms appear as a series of 43 to $56 \mathrm{kDa}$ bands due to differential $N$-glycosylation, with secreted IL-22BPi2 gaining $~ 8 \mathrm{kDa}$ (56 vs. $48 \mathrm{kDa}$ ) due to complex $N$-glycosylation.

SNP rs28385692, located in exon 2 of IL22RA2, changes the amino acid in position 16 of the protein from leucine to proline. This change occurs in the SP shared by the three IL-22BP isoforms produced by IL22RA2. In silico prediction methods diverged in their capacity to assign a functionally 
relevant effect on protein function to this variant. However, various in silico structural assessment methods coincided in predicting a shortening of the hydrophobic H-region of the IL-22BP SP by the 16P residue that may influence the precise location of the cleavage site with the mature portion of the IL-22BP proteins. Importantly, in this study, we went beyond the in silico predictions by assessing the effect of rs28385692 in vitro and showed experimentally using transfected cells that the p.Leu16Pro mutation significantly reduced the secreted levels of the IL-22BPi1, IL-22BPi2 and IL-22BPi3 isoforms. Specifically, the risk allele of p.Leu16Pro lowers secretion levels of each isoform to around $50 \%-60 \%$ of those of the respective wild-type isoforms as quantified using ELISA.

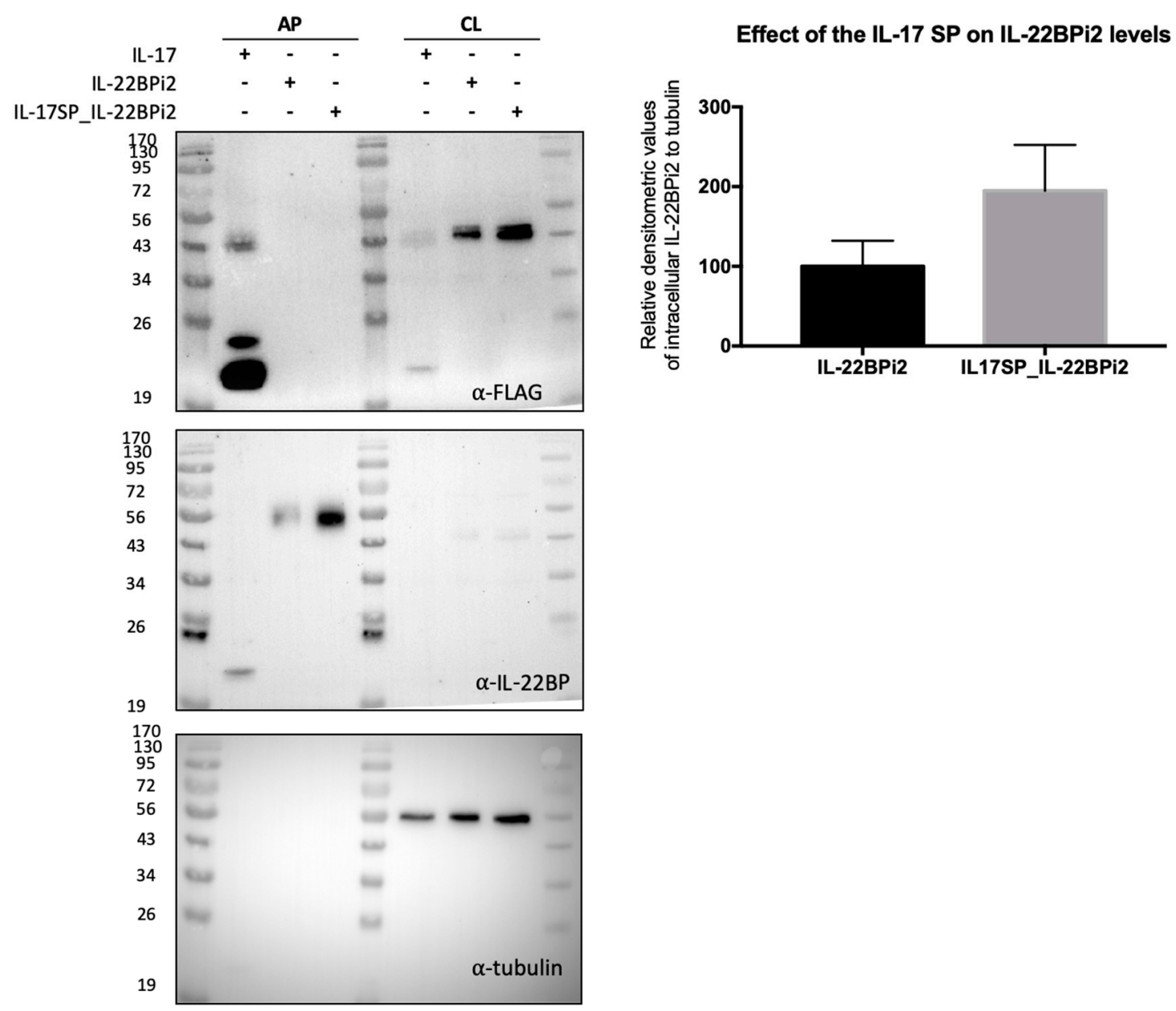

Figure 5. Native signal peptide of IL-22BP does not efficiently mediate secretion of IL-22BPi2. HEK293 cells were transfected with IL-17, IL-22BPi2, and IL-17SP_IL-22BPi2 expression plasmids, and $24 \mathrm{~h}$ later, cells were lysed and the conditioned medium subjected to acetone precipitation (AP). Both cell lysates (CL) and AP were resolved by SDS-PAGE under non-reducing conditions and immunoblotted against FLAG, IL-22BP and using tubulin as loading control. Intracellular IL-22BP reactive bands relative to tubulin ones were scanned and represented as fold change to IL-22BP wild-type (mean $\pm \mathrm{SD}, n=2$ ).

The literature documents several examples of similar changes in signal peptides that have been proven to affect secretion and are related to human diseases or traits: a Leu->Pro mutation in the signal peptide of COL5A1 (Alpha 1 Type V Collagen), a subunit of type V collagen, caused retention of this subunit in the endoplasmic reticulum and was associated with Ehlers-Danlos syndrome, a heritable connective tissue disease, in two unrelated families [45]. In addition, a common SNP causing a Leu->Pro mutation in the prohormone region of NPY (Neuropeptide Y) caused an increased secretion of this protein in chromaffin cells [46]. The inverse case, i.e., a change from proline to leucine, has also been reported to affect protein secretion: a Pro->Leu mutation in the signal peptide of DSPP 
(Dentin Sialophosphoprotein) resulted in a defective secretion of this protein and was associated with dentinogenesis imperfecta type III in a Korean family [47]. Finally, a Thr->Pro variant associated with coronary artery disease risk, located in the SP of the lysosomal acid lipase gene (LIPA), yields reduced LIPA protein levels and activity due to enhanced degradation [48].

The logistic regression analysis showed that associations of rs 28385692 and the main IL22RA2 GWAS SNP, rs17066096, with MS may be statistically independent. This suggests that there are at least two different variants causing association with MS within the IL22RA2 locus: one presumably corresponding to rs28385692 and the other one to a SNP in LD with rs17066096. rs17066096 itself is unlikely to be a causative variant, based on its intergenic location and its missing functional effect in our extensive in silico annotations. Recently, Lill and colleagues made an attempt to discover possible causal variants in LD with rs17066096. They performed an in silico and in vitro analysis of SNPs in the $3^{\prime}$ UTR of IL22RA2 that were at least in moderate LD with rs17066096 $\left(r^{2}>0.3\right)$ predicted to affect the binding of micro-RNAs (miRNAs; [49]). Although they successfully identified one SNP in a miRNA binding area, they did not find allele-specific differences on this binding; therefore, association of rs17066096 with MS remains unexplained. Despite these non-confirming functional data of Lill et al., rs17066063 might be a reasonable candidate for future functional studies given its location in a regulatory region of the gene.

Our study has several limitations. Firstly, in certain sub-analyses (e.g., individual populations), our study may have been underpowered to detect modest effects. Secondly, we restricted our analyses to individuals of European descent, which was self-reported. Thus, residual confounding due to undetected population stratification may have impacted some of our results and may account for some of the heterogeneity of the observed effect estimates. However, the effect size for the GWAS-derived SNP rs17066096 estimated in our datasets $(\mathrm{OR}=1.11)$ was very similar to the one described in the original GWAS (OR = 1.14) [4-6], suggesting that the impact of population substructure on our results is minor. Lastly, observing both genetic risk association and an effect of the genotypes on gene expression or quantitative protein production does not imply causality. Therefore, additional studies are necessary to characterize the exact molecular mechanisms of the IL22RA2 association signal in MS.

In summary, we successfully identified a comparatively rare genetic variant in IL22RA2 that is strongly - and likely independently of the primary GWAS signal-associated with MS. It causes an amino acid change in the signal peptide of IL-22BP and correspondingly, functional data generated for this study suggest an inhibitory effect on the trafficking of IL-22BP. Functional research on this variant will certainly provide insight about the role of this gene in MS and a better understanding of the still fairly unexplored function of IL-22BP isoforms in the immune system.

Author Contributions: Methodology, experimental design and execution, P.G.-F., A.L.d.L.P., J.M., A.U., I.A. (Iraide Alloza), I.A. (Ianire Astobiza), C.M.L., and K.V.; Validation, F.M., E.U., D.O., M.C., C.M.L., V.A., T.C.-T. and S.M.; Clinical and/or genetic data generation, A.A., T.C.-T., C.M.L., L.E.-P., X.M., M.C., O.A., M.B., A.C., B.F., P.-A.G., M.H., S.H., C.K., T.K., F.L., U.K.Z., F.Z. and K.V.; Formal analysis, A.L.d.L.P., V.A., P.G.-F. and K.V.; Writing-Original Draft Preparation, A.L.d.L.P., P.G.-F., I.A. (Iraide Alloza) and K.V.; Writing-Review and Editing, V.A., M.C., F.M., E.U., C.M.L., D.O. and K.V. with the help of all co-authors; Supervision, K.V. All authors have read and agreed to the published version of the manuscript.

Funding: This research was funded by Grupos de Investigación (IT512-10, PPG17/44), MINECO (Madrid, Spain; SAF2016-74891-R) and Red Española de Sclerosis Múltiple (REEM, ISCIII) RD16/0015/0005 to K.V, REEM RD16/0015/0004 to M.C., REEM RD/0015/0007 to D.O., REEM RD16/0015/0013 and FIS PI16/01259 to E.U., V.A. received funding from National Council for Scientific and Technological Development-CNPq (Brazil). C.M.L. received support by a "habilitation grant" (H01-2019) from the University of Lübeck.

Acknowledgments: We would like to thank Brit-Maren Schjeide for excellent technical support.

Conflicts of Interest: The authors declare no conflict of interest.

\section{Abbreviations}

$\mathrm{AP}$

CL

EAE
Acetone precipitate

Cell lysate

Experimental autoimmune encephalomyelitis 


$\begin{array}{ll}\text { EDSS } & \text { Expanded Disability Status Scale } \\ \text { GWAS } & \text { Genome-wide association screen } \\ \text { IL-22BPi1, 2 or 3 } & \text { Interleukin 22 binding protein isoform-1, -2 or -3 } \\ \text { IL22RA2 } & \text { Interleukin 22 receptor subunit alpha 2 } \\ \text { LD } & \text { Linkage disequilibrium } \\ \text { MAF } & \text { Minor allele frequency } \\ \text { OR } & \text { Odds ratio } \\ \text { PP } & \text { Primary progressive } \\ \text { RAF } & \text { Risk allele frequency } \\ \text { RR } & \text { Relapsing-remitting } \\ \text { ScP } & \text { Secondary progressive } \\ \text { SFM } & \text { Serum-free medium } \\ \text { SNP } & \text { Single nucleotide polymorphism } \\ \text { SP } & \text { Signal peptide }\end{array}$

\section{Appendix A}

Table A1. Association values of all SNPs included in the fine mapping conditioned to the index SNP, rs202573. ${ }^{1}$ OR: odds ratio. ${ }^{2} \mathrm{CI}$ : confidence interval.

\begin{tabular}{ccc}
\hline SNP & OR $^{\mathbf{1}} \mathbf{( 9 5}^{\mathbf{9}} \mathbf{C I}^{\mathbf{2}} \mathbf{)}$ & $\boldsymbol{p}$ \\
\hline rs4896239 & $0.95(0.798-1.131)$ & 0.5638 \\
rs17066096 & $1.059(0.8746-1.282)$ & 0.5569 \\
rs12194034 & $1.025(0.8412-1.249)$ & 0.8055 \\
rs1543509 & $0.9821(0.7719-1.25)$ & 0.8835 \\
rs28366 & $1.047(0.8594-1.275)$ & 0.6497 \\
rs276466 & $0.9779(0.7803-1.225)$ & 0.8458 \\
rs10484798 & $0.8452(0.6874-1.039)$ & 01105 \\
rs13217897 & $0.8249(0.6608-1.03)$ & 0.08906 \\
rs2064501 & $0.8337(0.6674-1.041)$ & 0.109 \\
rs11154914 & $1.043(0.7864-1.384)$ & 0.7687 \\
rs28385692 & $1.799(0.8654-3.739)$ & 0.1158 \\
rs13197049 & $0.846(0.6766-1.058)$ & 0.1423 \\
rs6570136 & $0.8669(0.7018-1.071)$ & 0.1855 \\
rs7745487 & $1.001(0.7436-1.347)$ & 0.9947 \\
\hline
\end{tabular}

Table A2. Association values of the haplotypes in blocks. LD blocks were calculated with the confidence interval method using Haploview $[25,26]$.

\begin{tabular}{ccccc}
\hline Block & Haplotype & Frequency & Case, Control Frequencies & $p$ \\
\hline \multirow{2}{*}{ rs4896239+ rs28385692 } & TA & 0.501 & $0.479,0.519$ & 0.102 \\
& CG & 0.267 & $0.273,0.262$ & 0.6031 \\
& CA & 0.232 & $0.248,0.219$ & 0.1642 \\
\hline rs12194034+ & TTTA & 0.408 & $0.404,0.411$ & 0.763 \\
rs1543509+rs28366+ & TTTG & 0.22 & $0.216,0.224$ & 0.7026 \\
rs276466 & ATCA & 0.217 & $0.215,0.218$ & 0.8637 \\
& TCTA & 0.148 & $0.160,0.139$ & 0.2266 \\
\hline rs132117897+ rs202573 & GGCAA & 0.495 & $0.475,0.512$ & 0.1298 \\
+ rs2064501 + & AGTAT & 0.193 & $0.178,0.206$ & 0.1565 \\
rs11154914+ & GATGA & 0.172 & $0.192,0.156$ & 0.0561 \\
rs13197049 & GATAA & 0.124 & $0.139,0.111$ & 0.0806 \\
\hline \multirow{2}{*}{ rs6570136+rs7745487 } & GG & 0.551 & $0.543,0.558$ & 0.5396 \\
& AG & 0.278 & $0.265,0.289$ & 0.2853 \\
& AA & 0.171 & $0.192,0.153$ & 0.0373 \\
\hline
\end{tabular}


Table A3. Features of the individual and consensus computational tools used in this study. Adapted from [42].

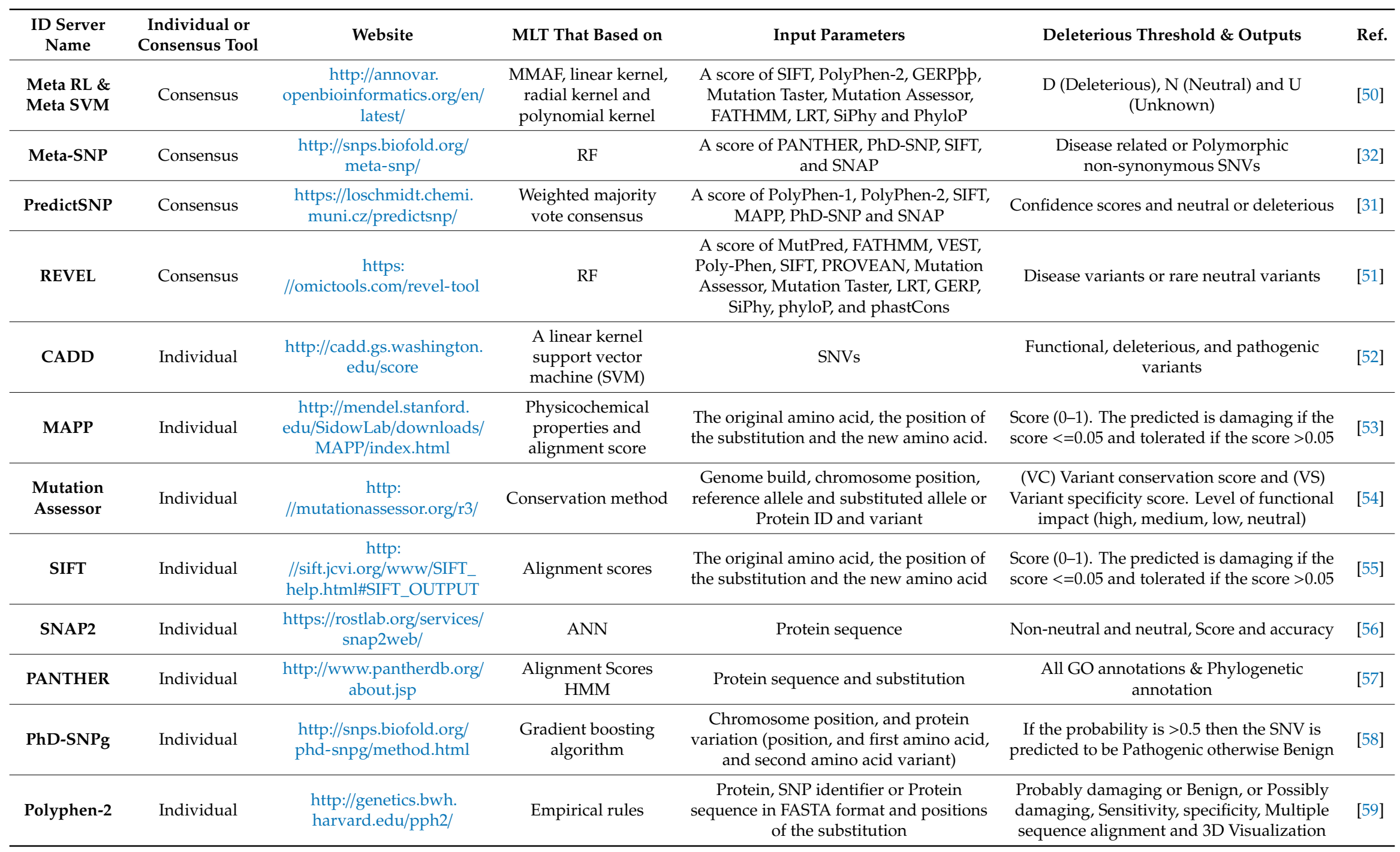



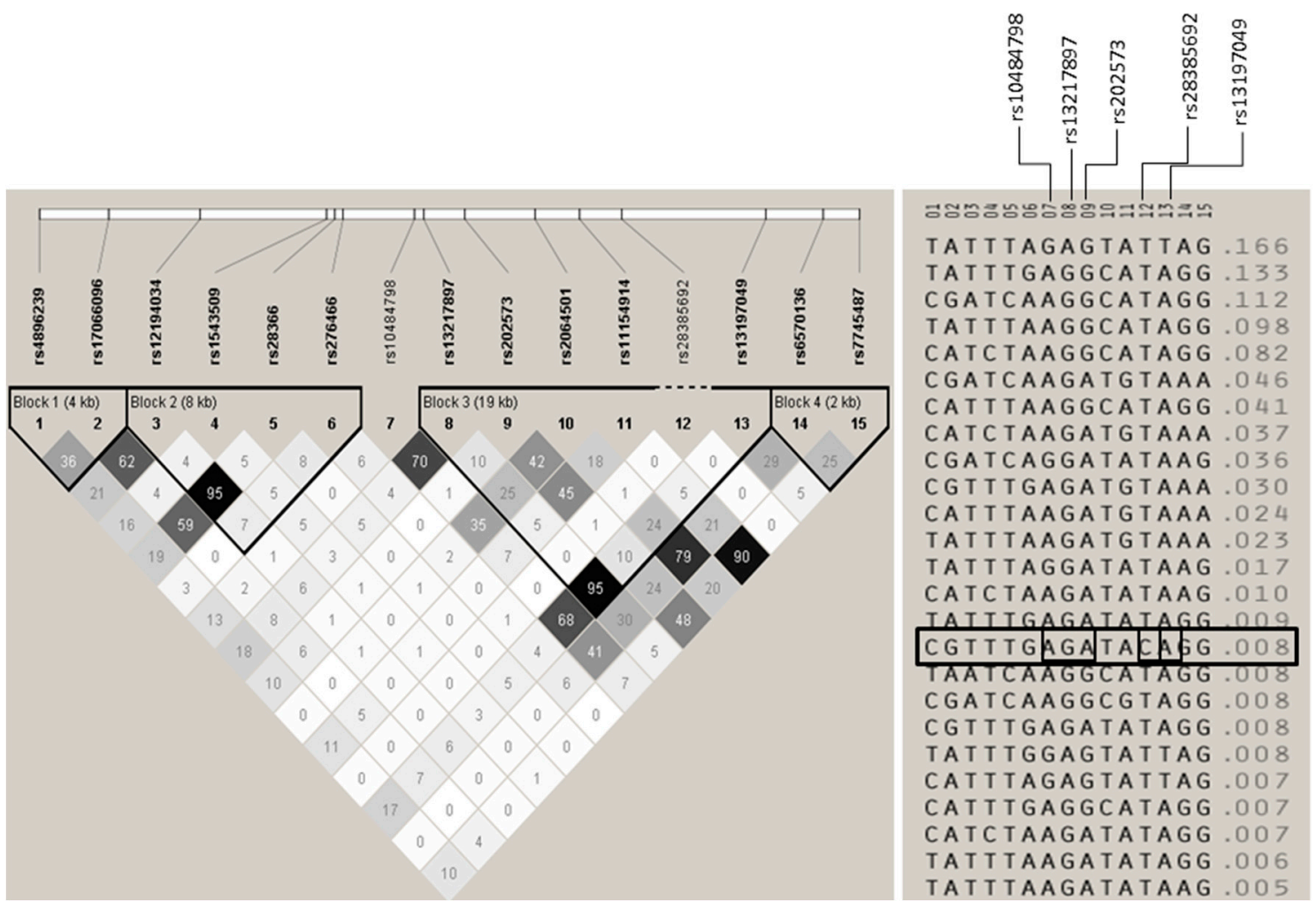

Figure A1. Linkage disequilibrium structure and haplotypes of IL22RA2. On the left, LD plot of the 15 SNPs analyzed in the Bilbao cohort. Blocks were calculated using the confidence interval algorithm implemented in Haploview $[25,26]$. Only samples genotyped for all SNPs were considered for the haplotype calculation. The numbers inside the squares indicate $r^{2}$ values, and darker shades of gray represent higher degrees of linkage disequilibrium. On the right, all the haplotypes that were present in the Bilbao cohorts considering all SNPs in a single LD block are represented. The only haplotype containing the risk (C) allele of the non-synonymous SNP rs28385692 is boxed. SNPs with significant associations and rs28385692 are indicated on top of the right panel.

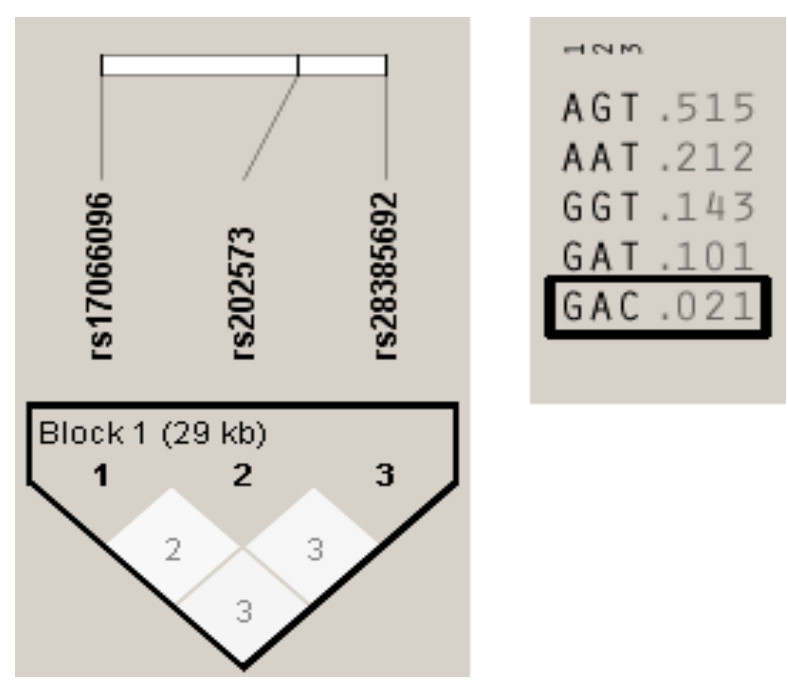

Figure A2. Haplotypes formed by the three SNPs genotyped in the Basque Country, Madrid, Andalucía, Barcelona and Germany populations. The three SNPs were considered in a single haplotype block. On the left, LD plot showing pairwise $r^{2}$ values between the SNPs. On the right, the 5 SNP haplotypes present in the combined cohorts. The only haplotype containing the C allele of rs28385692 is boxed. 
(a) PredictSNP

\begin{tabular}{|c|c|c|c|c|c|c|c|c|c|}
\hline \multicolumn{3}{|l|}{ RESULTS } & \multicolumn{2}{|c|}{ neutral } & deleterious & \multicolumn{3}{|c|}{ XX \% expected accuracy } & \multirow[b]{2}{*}{ PANTHER } \\
\hline Annotation & Mutation & PredictSNP & MAPP & PhD-SNP & PolyPhen-1 & PolyPhen-2 & SIFT & SNAP & \\
\hline IL-22BPi1 & L16P & $55 \%$ & $75 \%$ & $59 \%$ & $67 \%$ & $\cdot$ & $70 \%$ & $89 \%$ & $68 \%$ \\
\hline IL-22BPi2 & L16P & $61 \%$ & $76 \%$ & $61 \%$ & $59 \%$ & $63 \%$ & $68 \%$ & $89 \%$ & $68 \%$ \\
\hline IL-22BPi3 & L16P & $51 \%$ & $76 \%$ & $61 \%$ & $59 \%$ & $63 \%$ & $74 \%$ & $55 \%$ & $68 \%$ \\
\hline
\end{tabular}

(b) Meta-SNP

\begin{tabular}{|c|c|c|c|c|c|c|c|c|}
\hline \multirow[b]{2}{*}{ IL-22BPi1 } & Mutation & PANTHER & PhD-SNP & SIFT & SNAP & Meta-SNP & RI & \multirow{5}{*}{ 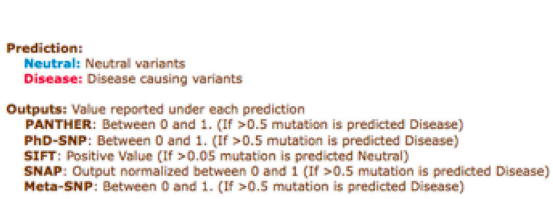 } \\
\hline & L16P & $\begin{array}{r}\text { Neutral } \\
0.283\end{array}$ & $\begin{array}{r}\text { Disease } \\
0.672\end{array}$ & $\begin{array}{r}\text { Neutral } \\
0.090\end{array}$ & $\begin{array}{r}\text { Disease } \\
0.750\end{array}$ & $\begin{array}{r}\text { Neutral } \\
0.432\end{array}$ & 1 & \\
\hline \multirow{3}{*}{ IL-22BPi2 } & Mutation & PANTHER & PhD-SNP & SIFT & SNAP & Meta-SNP & RI & \\
\hline & L16P & $\begin{array}{r}\text { Neutral } \\
0.283\end{array}$ & $\begin{array}{r}\text { Disease } \\
0.696\end{array}$ & $\begin{array}{r}\text { Neutral } \\
0.290\end{array}$ & $\begin{array}{r}\text { Disease } \\
0.650\end{array}$ & $\begin{array}{c}\text { Neutral } \\
0.493\end{array}$ & 0 & \\
\hline & Mutation & PANTHER & PhD-SNP & SIFT & SNAP & Meta-SNP & RI & \\
\hline IL-22BPi3 & L16P & $\begin{array}{r}\text { Neutral } \\
0.283\end{array}$ & $\begin{array}{r}\text { Disease } \\
0.686\end{array}$ & $\begin{array}{r}\text { Neutral } \\
0.240\end{array}$ & $\begin{array}{r}\text { Disease } \\
0.700\end{array}$ & $\begin{array}{r}\text { Neutral } \\
0.466\end{array}$ & 1 & RI: Reliability Index between 0 and 10 \\
\hline
\end{tabular}

(c) Ensemble

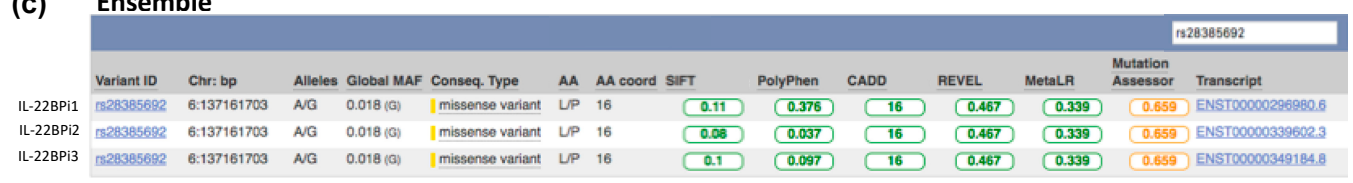

Figure A3. The pathogenicity prediction of rs28385692 using PredictSNP (a), Meta-SNP (b), and (c) Ensemble VEP web servers. The results indicate the predictive severity of the p.Leu16Pro variant conferred by the SNP rs28385692 for the three IL-22BP isoforms.

IL-22BP 16L

(a) SignalP 5.0

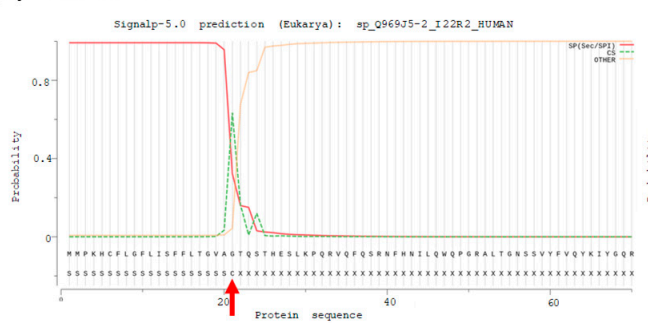

(b) Predisi

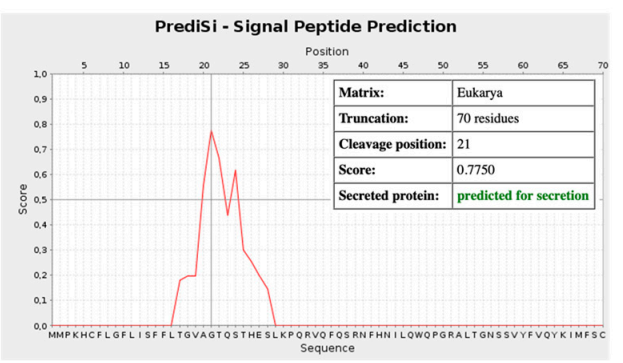

(c) Signal-3L

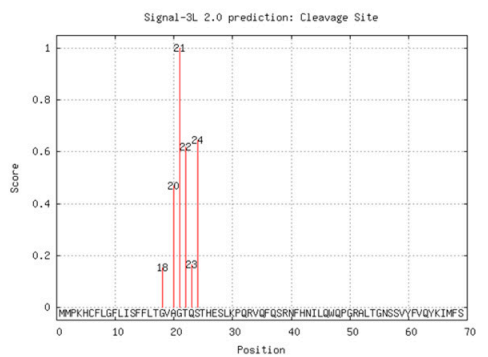

IL-22BP 16P
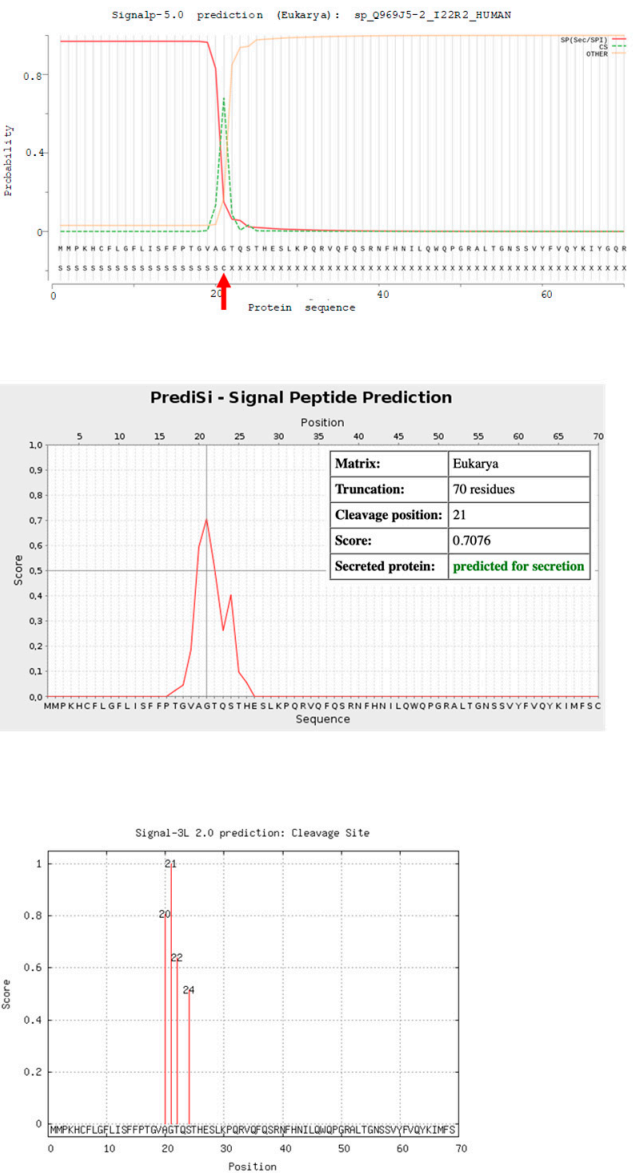

Figure A4. Cont. 
(d) RaptorX
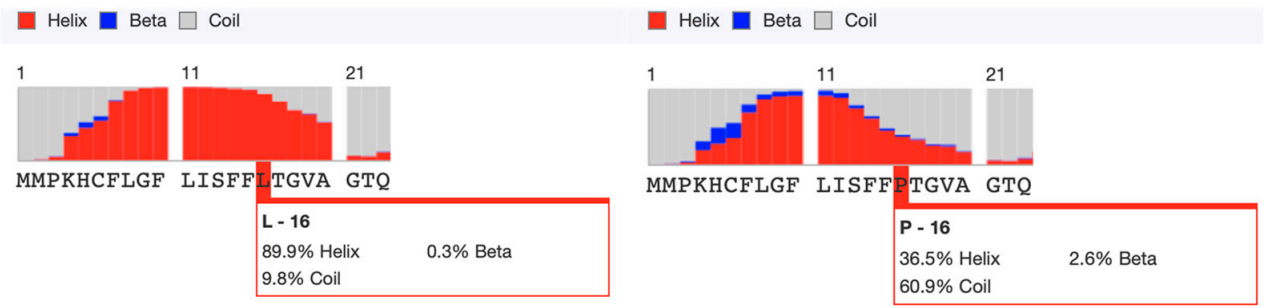

(e) SABLE
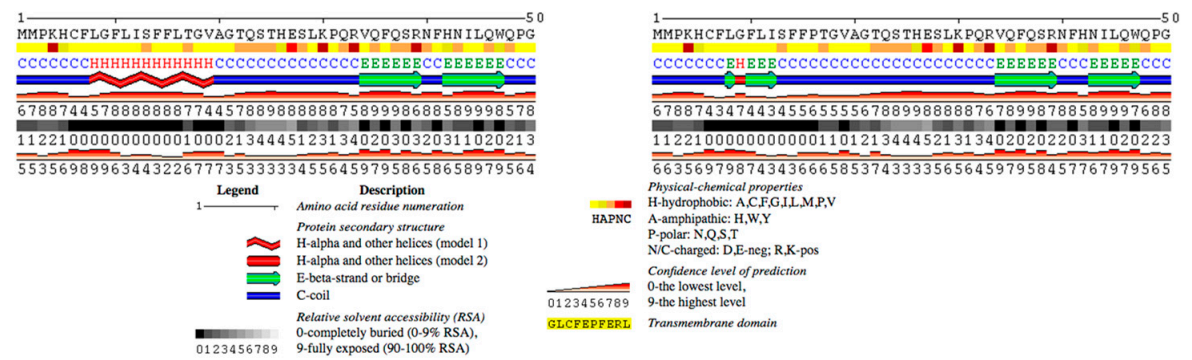

Figure A4. Signal peptide prediction in the Leu16 and Pro16 variant IL-22BP proteins. SignalP 5.0, PrediSi and Signal-3L predicted the cleavage site for both wild-type and p.Leu16Pro mutant to be the 21st residue of the signal peptide. Panels $(\mathbf{a}-\mathbf{c})$ show cleavage sites from SignalP 5.0, PrediSi and Signal-3L, respectively. Panels $(\mathbf{d}, \mathbf{e})$ represent the secondary structure predictions from RaptorX and SABLE, respectively.

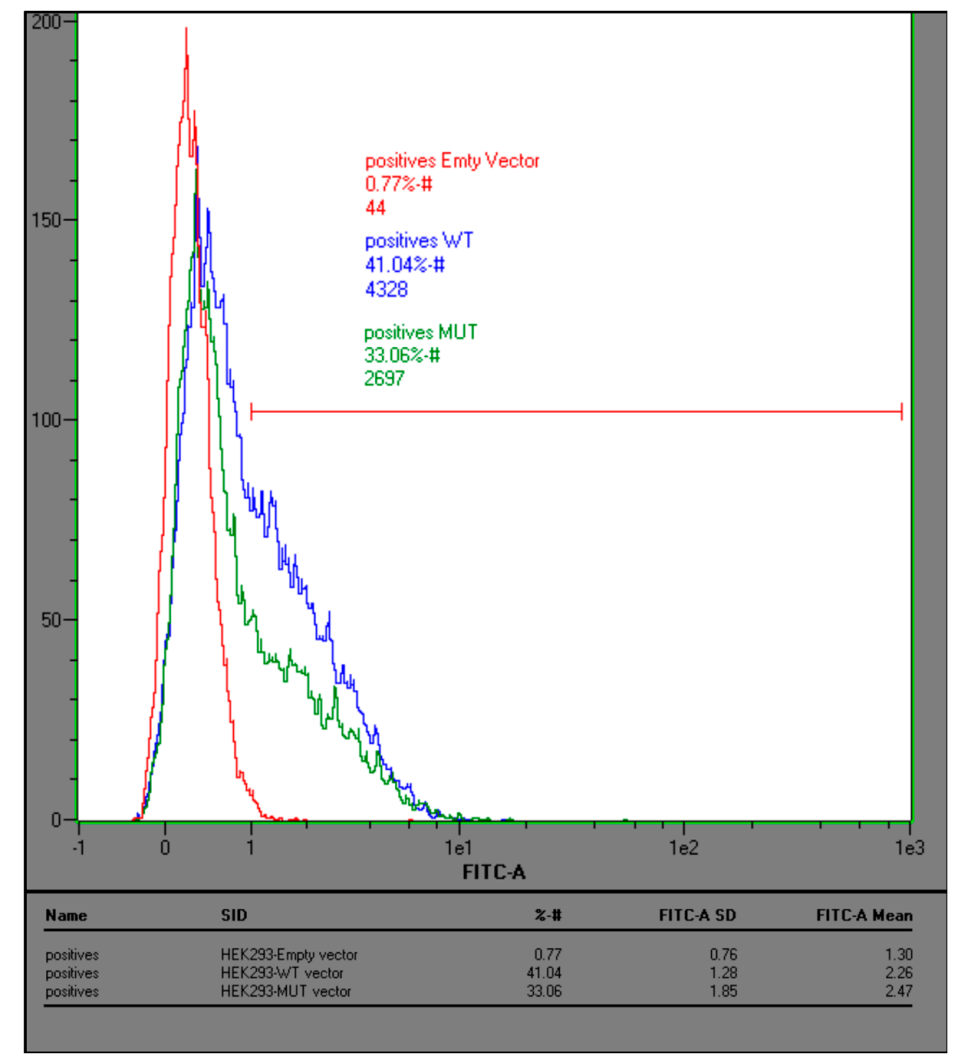

Figure A5. Leu16 to Pro mutation in the signal peptide of IL-22BPi2 is associated with lower intracellular levels. HEK293 cells were transfected with the IL22RA2v2 wild type (Leu16), mutant (16P) or empty expression plasmids; $24 \mathrm{~h}$ later cells were fixed, permeabilized and immunostained with anti-IL22BP antibody. Cells were analyzed by flow cytometry. 


\section{References}

1. Patsopoulos, N.A.; Baranzini, S.E.; Santaniello, A.; Shoostari, P.; Cotsapas, C.; Wong, G.; Beecham, A.H.; James, T.; Replogle, J.; Vlachos, I.S.; et al. Multiple sclerosis genomic map implicates peripheral immune cells and microglia in susceptibility. Science 2019, 365, eaav7188. [CrossRef]

2. Beyeen, A.D.; Adzemovic, M.Z.; Ockinger, J.; Stridh, P.; Becanovic, K.; Laaksonen, H.; Lassmann, H.; Harris, R.A.; Hillert, J.; Alfredsson, L.; et al. IL-22RA2 Associates with Multiple Sclerosis and Macrophage Effector Mechanisms in Experimental Neuroinflammation. J. Immunol. 2010, 185, 6883-6890. [CrossRef] [PubMed]

3. Vandenbroeck, K.; Alvarez, J.; Swaminathan, B.; Alloza, I.; Matesanz, F.; Urcelay, E.; Comabella, M.; Alcina, A.; Fedetz, M.; Ortiz, M.A.; et al. A cytokine gene screen uncovers SOCS1 as genetic risk factor for multiple sclerosis. Genes Immun. 2012, 13, 21-28. [CrossRef] [PubMed]

4. Beecham, A.H.; Patsopoulos, N.A.; Xifara, D.K.; Davis, M.F.; Kemppinen, A.; Cotsapas, C.; Shah, T.S.; Spencer, C.; Booth, D.; Goris, A.; et al. Analysis of immune-related loci identifies 48 new susceptibility variants for multiple sclerosis. Nat. Genet. 2013, 45, 1353-1362. [PubMed]

5. Bush, W.S.; Sawcer, S.J.; De Jager, P.L.; The International Multiple Sclerosis Genetics Consortium (IMSGC). A systems biology approach uncovers cell-specific gene regulatory effects of genetic associations in multiple sclerosis. Am. J. Nat. Commun. 2019, 10, 2236. [CrossRef]

6. Sawcer, S.; Hellenthal, G.; Pirinen, M.; Spencer, C.C.A.; Patsopoulos, N.A.; Moutsianas, L.; Dilthey, A.; Su, Z.; Freeman, C.; Hunt, S.E.; et al. Genetic risk and a primary role for cell-mediated immune mechanisms in multiple sclerosis. Nature 2011, 476, 214-219. [PubMed]

7. Wolk, K.; Kunz, S.; Witte, E.; Friedrich, M.; Asadullah, K.; Sabat, R. IL-22 Increases the Innate Immunity of Tissues. Immunity 2004, 21, 241-254. [CrossRef]

8. Wolk, K.; Sabat, R. Interleukin-22: A novel T- and NK-cell derived cytokine that regulates the biology of tissue cells. Cytokine Growth Factor Rev. 2006, 17, 367-380. [CrossRef]

9. Laaksonen, H.; Guerreiro-Cacais, A.O.; Adzemovic, M.Z.; Parsa, R.; Zeitelhofer, M.; Jagodic, M.; Olsson, T. The multiple sclerosis risk gene IL22RA2 contributes to a more severe murine autoimmune neuroinflammation. Genes Immun. 2014, 15, 457-465. [CrossRef]

10. Kebir, H.; Kreymborg, K.; Ifergan, I.; Dodelet-Devillers, A.; Cayrol, R.; Bernard, M.; Giuliani, F.; Arbour, N.; Becher, B.; Prat, A. Human TH17 lymphocytes promote blood-brain barrier disruption and central nervous system inflammation. Nat. Med. 2007, 13, 1173-1175. [CrossRef]

11. Almolda, B.; Costa, M.; Montoya, M.; González, B.; Castellano, B. Increase in Th17 and T-reg Lymphocytes and Decrease of IL22 Correlate with the Recovery Phase of Acute EAE IN Rat. PLoS ONE 2011, 6, e27473. [CrossRef] [PubMed]

12. Xu, W.; Li, R.; Dai, Y.; Wu, A.; Wang, H.; Cheng, C.; Qiu, W.; Lu, Z.; Zhong, X.; Shu, Y.; et al. IL-22 secreting CD4+ T cells in the patients with neuromyelitis optica and multiple sclerosis. J. Neuroimmunol. 2013, 261, 87-91. [CrossRef] [PubMed]

13. Perriard, G.; Mathias, A.; Enz, L.; Canales, M.; Schluep, M.; Gentner, M.; Schaeren-Wiemers, N.; Du Pasquier, R.A. Interleukin-22 is increased in multiple sclerosis patients and targets astrocytes. J. Neuroinflammation 2015, 12, 119. [CrossRef] [PubMed]

14. Kotenko, S.V.; Izotova, L.S.; Mirochnitchenko, O.V.; Esterova, E.; Dickensheets, H.; Donnelly, R.P.; Pestka, S. Identification, Cloning, and Characterization of a Novel Soluble Receptor That Binds IL-22 and Neutralizes Its Activity. J. Immunol. 2001, 166, 7096-7103. [CrossRef] [PubMed]

15. Dumoutier, L.; Lejeune, D.; Colau, D.; Renauld, J.-C. Cloning and Characterization of IL-22 Binding Protein, a Natural Antagonist of IL-10-Related T Cell-Derived Inducible Factor/IL-22. J. Immunol. 2001, 166, 7090-7095. [CrossRef] [PubMed]

16. Jones, B.C.; Logsdon, N.J.; Walter, M.R. Structure of IL-22 Bound to Its High-Affinity IL-22R1 Chain. Structure 2008, 16, 1333-1344. [CrossRef] [PubMed]

17. Logsdon, N.J.; Jones, B.C.; Josephson, K.; Cook, J.; Walter, M.R. Comparison of Interleukin-22 and Interleukin-10 Soluble Receptor Complexes. J. Interf. Cytokine Res. 2002, 22, 1099-1112. [CrossRef] [PubMed] 
18. Wolk, K.; Witte, E.; Hoffmann, U.; Doecke, W.-D.W.-D.W.-D.; Endesfelder, S.; Asadullah, K.; Sterry, W.; Volk, H.-D.H.-D.H.-D.; Wittig, B.M.; Sabat, R. IL-22 Induces Lipopolysaccharide-Binding Protein in Hepatocytes: A Potential Systemic Role of IL-22 in Crohn's Disease. J. Immunol. 2007, 178, 5973-5981. [CrossRef]

19. Lim, C.; Hong, M.; Savan, R. Human IL-22 binding protein isoforms act as a rheostat for IL-22 signaling. Sci. Signal. 2016, 9, ra95. [CrossRef]

20. Gómez-Fernández, P.; Urtasun, A.; Paton, A.W.; Paton, J.C.; Borrego, F.; Dersh, D.; Argon, Y.; Alloza, I.; Vandenbroeck, K. Long Interleukin-22 Binding Protein Isoform-1 Is an Intracellular Activator of the Unfolded Protein Response. Front. Immunol. 2018, 9, 2934. [CrossRef]

21. McDonald, W.I.; Compston, A.; Edan, G.; Goodkin, D.; Hartung, H.P.; Lublin, F.D.; McFarland, H.F.; Paty, D.W.; Polman, C.H.; Reingold, S.C.; et al. Recommended diagnostic criteria for multiple sclerosis: Guidelines from the International Panel on the diagnosis of multiple sclerosis. Ann. Neurol. 2001, 50, 121-127. [CrossRef] [PubMed]

22. Poser, C.M.; Paty, D.W.; Scheinberg, L.; McDonald, W.I.; Davis, F.A.; Ebers, G.C.; Johnson, K.P.; Sibley, W.A.; Silberberg, D.H.; Tourtellotte, W.W. New diagnostic criteria for multiple sclerosis: Guidelines for research protocols. Ann. Neurol. 1983, 13, 227-231. [CrossRef] [PubMed]

23. Purcell, S.; Cherny, S.S.; Sham, P.C. Genetic Power Calculator: Design of linkage and association genetic mapping studies of complex traits. Bioinformatics 2003, 19, 149-150. [CrossRef] [PubMed]

24. Purcell, S.; Neale, B.; Todd-Brown, K.; Thomas, L.; Ferreira, M.A.R.; Bender, D.; Maller, J.; Sklar, P.; de Bakker, P.I.W.; Daly, M.J.; et al. PLINK: A Tool Set for Whole-Genome Association and Population-Based Linkage Analyses. Am. J. Hum. Genet. 2007, 81, 559-575. [CrossRef]

25. Barrett, J.C.; Fry, B.; Maller, J.; Daly, M.J. Haploview: Analysis and visualization of LD and haplotype maps. Bioinformatics 2005, 21, 263-265. [CrossRef]

26. Gabriel, S.B.; Schaffner, S.F.; Nguyen, H.; Moore, J.M.; Roy, J.; Blumenstiel, B.; Higgins, J.; DeFelice, M.; Lochner, A.; Faggart, M.; et al. The structure of haplotype blocks in the human genome. Science 2002, 296, 2225-2229. [CrossRef]

27. Skol, A.D.; Scott, L.J.; Abecasis, G.R.; Boehnke, M. Joint analysis is more efficient than replication-based analysis for two-stage genome-wide association studies. Nat. Genet. 2006, 38, 209-213. [CrossRef]

28. Johnson, A.D.; Handsaker, R.E.; Pulit, S.L.; Nizzari, M.M.; O’Donnell, C.J.; de Bakker, P.I.W. SNAP: A web-based tool for identification and annotation of proxy SNPs using HapMap. Bioinformatics 2008, 24, 2938-2939. [CrossRef]

29. McLaren, W.; Gil, L.; Hunt, S.E.; Riat, H.S.; Ritchie, G.R.S.; Thormann, A.; Flicek, P.; Cunningham, F. The Ensembl Variant Effect Predictor. Genome Biol. 2016, 17, 122. [CrossRef]

30. Boyle, A.P.; Hong, E.L.; Hariharan, M.; Cheng, Y.; Schaub, M.A.; Kasowski, M.; Karczewski, K.J.; Park, J.; Hitz, B.C.; Weng, S.; et al. Annotation of functional variation in personal genomes using RegulomeDB. Genome Res. 2012, 22, 1790-1797. [CrossRef]

31. Bendl, J.; Stourac, J.; Salanda, O.; Pavelka, A.; Wieben, E.D.; Zendulka, J.; Brezovsky, J.; Damborsky, J. PredictSNP: Robust and Accurate Consensus Classifier for Prediction of Disease-Related Mutations. PLoS Comput. Biol. 2014, 10, e1003440. [CrossRef] [PubMed]

32. Capriotti, E.; Altman, R.B.; Bromberg, Y. Collective judgment predicts disease-associated single nucleotide variants. BMC Genomics 2013, 14, S2. [CrossRef] [PubMed]

33. Cunningham, F.; Achuthan, P.; Akanni, W.; Allen, J.; Amode, M.R.; Armean, I.M.; Bennett, R.; Bhai, J.; Billis, K.; Boddu, S.; et al. Ensembl 2019. Nucleic Acids Res. 2019, 47, D745-D751. [CrossRef] [PubMed]

34. Bendtsen, J.D.; Nielsen, H.; von Heijne, G.; Brunak, S. Improved prediction of signal peptides: SignalP 3.0. J. Mol. Biol. 2004, 340, 783-795. [CrossRef] [PubMed]

35. Kall, L.; Krogh, A.; Sonnhammer, E.L.L. Advantages of combined transmembrane topology and signal peptide prediction-The Phobius web server. Nucleic Acids Res. 2007, 35, W429-W432. [CrossRef] [PubMed]

36. Buchan, D.W.A.; Jones, D.T. The PSIPRED Protein Analysis Workbench: 20 years on. Nucleic Acids Res. 2019, 47, 4068-4085. [CrossRef]

37. Almagro Armenteros, J.J.; Tsirigos, K.D.; Sønderby, C.K.; Petersen, T.N.; Winther, O.; Brunak, S.; von Heijne, G.; Nielsen, H. SignalP 5.0 improves signal peptide predictions using deep neural networks. Nat. Biotechnol. 2019, 37, 420-423. [CrossRef] 
38. Hiller, K.; Grote, A.; Scheer, M.; Munch, R.; Jahn, D. PrediSi: Prediction of signal peptides and their cleavage positions. Nucleic Acids Res. 2004, 32, W375-W379. [CrossRef]

39. Buchan, D.W.A.A.; Minneci, F.; Nugent, T.C.O.O.; Bryson, K.; Jones, D.T. Scalable web services for the PSIPRED Protein Analysis Workbench. Nucleic Acids Res. 2013, 41, 349-357. [CrossRef]

40. Wang, S.; Li, W.; Liu, S.; Xu, J. RaptorX-Property: A web server for protein structure property prediction. Nucleic Acids Res. 2016, 44, W430-W435. [CrossRef]

41. Adamczak, R.; Porollo, A.; Meller, J. Accurate prediction of solvent accessibility using neural networks-based regression. Proteins Struct. Funct. Bioinform. 2004, 56, 753-767. [CrossRef] [PubMed]

42. Hassan, M.S.; Shaalan, A.A.; Dessouky, M.I.; Abdelnaiem, A.E.; Abdel-Haleem, D.A.; ElHefnawi, M. Predicting Non-Synonymous Single Nucleotide Variants Pathogenic Effects in Human Diseases. In Encyclopedia of Bioinformatics and Computational Biology; Elsevier Ltd.: Amserdam, The Netherlands, 2018; pp. 400-409. ISBN 9780128096338.

43. Owji, H.; Nezafat, N.; Negahdaripour, M.; Hajiebrahimi, A.; Ghasemi, Y. A comprehensive review of signal peptides: Structure, roles, and applications. Eur. J. Cell Biol. 2018, 97, 422-441. [CrossRef] [PubMed]

44. Hegde, R.S.; Bernstein, H.D. The surprising complexity of signal sequences. Trends Biochem. Sci. 2006, 31, 563-571. [CrossRef] [PubMed]

45. Symoens, S.; Malfait, F.; Renard, M.; André, J.; Hausser, I.; Loeys, B.; Coucke, P.; De Paepe, A. COL5A1 signal peptide mutations interfere with protein secretion and cause classic Ehlers-Danlos syndrome. Hum. Mutat. 2009, 30, 395-403. [CrossRef] [PubMed]

46. Mitchell, G.C.; Wang, Q.; Ramamoorthy, P.; Whim, M.D. A Common Single Nucleotide Polymorphism Alters the Synthesis and Secretion of Neuropeptide Y. J. Neurosci. 2008, 28, 14428-14434. [CrossRef]

47. Lee, S.-K.; Lee, K.-E.; Song, S.J.; Hyun, H.-K.; Lee, S.-H.; Kim, J.-W. A DSPP Mutation Causing Dentinogenesis Imperfecta and Characterization of the Mutational Effect. Biomed. Res. Int. 2013, 2013, 1-7.

48. Morris, G.E.; Braund, P.S.; Moore, J.S.; Samani, N.J.; Codd, V.; Webb, T.R. Coronary Artery Disease-Associated LIPA Coding Variant rs1051338 Reduces Lysosomal Acid Lipase Levels and Activity in Lysosomes. Arterioscler. Thromb. Vasc. Biol. 2017, 37, 1050-1057. [CrossRef]

49. Lill, C.M.; Schilling, M.; Ansaloni, S.; Schröder, J.; Jaedicke, M.; Luessi, F.; Schjeide, B.-M.M.; Mashychev, A.; Graetz, C.; Akkad, D.A.; et al. Assessment of microRNA-related SNP effects in the $3^{\prime}$ untranslated region of the IL22RA2 risk locus in multiple sclerosis. Neurogenetics 2014, 15, 129-134. [CrossRef]

50. Dong, C.; Wei, P.; Jian, X.; Gibbs, R.; Boerwinkle, E.; Wang, K.; Liu, X. Comparison and integration of deleteriousness prediction methods for nonsynonymous SNVs in whole exome sequencing studies. Hum. Mol. Genet. 2015, 24, 2125-2137. [CrossRef]

51. Ioannidis, N.M.; Rothstein, J.H.; Pejaver, V.; Middha, S.; McDonnell, S.K.; Baheti, S.; Musolf, A.; Li, Q.; Holzinger, E.; Karyadi, D.; et al. REVEL: An Ensemble Method for Predicting the Pathogenicity of Rare Missense Variants. Am. J. Hum. Genet. 2016, 99, 877-885. [CrossRef]

52. Rentzsch, P.; Witten, D.; Cooper, G.M.; Shendure, J.; Kircher, M. CADD: Predicting the deleteriousness of variants throughout the human genome. Nucleic Acids Res. 2019, 47, D886-D894. [CrossRef] [PubMed]

53. Stone, E.A.; Sidow, A. Physicochemical constraint violation by missense substitutions mediates impairment of protein function and disease severity. Genome Res. 2005, 15, 978-986. [CrossRef] [PubMed]

54. Reva, B.; Antipin, Y.; Sander, C. Predicting the functional impact of protein mutations: Application to cancer genomics. Nucleic Acids Res. 2011, 39, e118. [CrossRef] [PubMed]

55. Kumar, P.; Henikoff, S.; Ng, P.C. Predicting the effects of coding non-synonymous variants on protein function using the SIFT algorithm. Nat. Protoc. 2009, 4, 1073-1081. [CrossRef]

56. Bromberg, Y.; Rost, B. SNAP: Predict effect of non-synonymous polymorphisms on function. Nucleic Acids Res. 2007, 35, 3823-3835. [CrossRef]

57. Thomas, P.D.; Kejariwal, A. Coding single-nucleotide polymorphisms associated with complex vs. Mendelian disease: Evolutionary evidence for differences in molecular effects. Proc. Natl. Acad. Sci. USA 2004, 101, 15398-15403. [CrossRef] 
58. Capriotti, E.; Calabrese, R.; Casadio, R. Predicting the insurgence of human genetic diseases associated to single point protein mutations with support vector machines and evolutionary information. Bioinformatics 2006, 22, 2729-2734. [CrossRef]

59. Adzhubei, I.A.; Schmidt, S.; Peshkin, L.; Ramensky, V.E.; Gerasimova, A.; Bork, P.; Kondrashov, A.S.; Sunyaev, S.R. A method and server for predicting damaging missense mutations. Nat. Methods 2010, 7 , 248-249. [CrossRef]

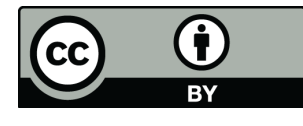

(C) 2020 by the authors. Licensee MDPI, Basel, Switzerland. This article is an open access article distributed under the terms and conditions of the Creative Commons Attribution (CC BY) license (http://creativecommons.org/licenses/by/4.0/). 